\title{
Error analysis of a mixed finite element method for the Cahn- Hilliard equation
}

\section{Journal Article}

Author(s):

Feng, Xiaobing; Prohl, Andreas

Publication date:

2004-11

Permanent link:

https://doi.org/10.3929/ethz-b-000038267

Rights / license:

In Copyright - Non-Commercial Use Permitted

Originally published in:

Numerische Mathematik 99(1), https://doi.org/10.1007/s00211-004-0546-5 


\title{
Error analysis of a mixed finite element method for the Cahn-Hilliard equation
}

\author{
Xiaobing Feng ${ }^{1}$, Andreas Prohl ${ }^{2}$ \\ 1 Department of Mathematics, The University of Tennessee, Knoxville, TN 37996, USA; \\ e-mail: xfeng@math.utk.edu \\ 2 Department of Mathematics, ETH Zurich, 8092 Zurich, Switzerland; \\ e-mail: apr@math.ethz.ch
}

Received March 4, 2003 / Revised version received May 7, 2004

Published online September 6, 2004 - (c) Springer-Verlag 2004

Summary. We propose and analyze a semi-discrete and a fully discrete mixed finite element method for the Cahn-Hilliard equation $u_{t}+\Delta(\varepsilon \Delta u-$ $\left.\varepsilon^{-1} f(u)\right)=0$, where $\varepsilon>0$ is a small parameter. Error estimates which are quasi-optimal order in time and optimal order in space are shown for the proposed methods under minimum regularity assumptions on the initial data and the domain. In particular, it is shown that all error bounds depend on $\frac{1}{\varepsilon}$ only in some lower polynomial order for small $\varepsilon$. The cruxes of our analysis are to establish stability estimates for the discrete solutions, to use a spectrum estimate result of Alikakos and Fusco [2], and Chen [15] to prove a discrete counterpart of it for a linearized Cahn-Hilliard operator to handle the nonlinear term on a stretched time grid. The ideas and techniques developed in this paper also enable us to prove convergence of the fully discrete finite element solution to the solution of the Hele-Shaw (Mullins-Sekerka) problem as $\varepsilon \rightarrow 0$ in [29].

Mathematics Subject Classification (1991): 65M60, 65M12, 65M15, 35B25, 35K57, 35Q99, 53A10

Correspondence to: A. Prohl 


\section{Introduction}

In this paper we consider the following Cahn-Hilliard problem:

$$
\begin{aligned}
u_{t}+\Delta\left(\varepsilon \Delta u-\frac{1}{\varepsilon} f(u)\right) & =0 & & \text { in } \Omega_{T}:=\Omega \times(0, T), \\
\frac{\partial u}{\partial n}=\frac{\partial}{\partial n}\left(\varepsilon \Delta u-\frac{1}{\varepsilon} f(u)\right) & =0 & & \text { in } \partial \Omega_{T}:=\partial \Omega \times(0, T), \\
u & =u_{0} & & \text { in } \Omega \times\{0\} .
\end{aligned}
$$

Here $\Omega \subset \mathbf{R}^{N}(N=2,3)$ is a bounded domain, $T>0$ is a fixed constant, and $f$ is the derivative of a smooth double equal well potential taking its global minimum value 0 at $u= \pm 1$. A typical example of $f$ is

$$
f(u):=F^{\prime}(u) \quad \text { and } \quad F(u)=\frac{1}{4}\left(u^{2}-1\right)^{2} .
$$

The equation (1) was originally introduced by Cahn and Hilliard [12] to describe the complicated phase separation and coarsening phenomena in a solid where only two different concentration phases can exist stably. Note that the equation (1) differs from the original Cahn-Hilliard equation (see [12]) in the scaling of the time so that $t$ here, called the fast time, represents $\frac{t}{\varepsilon}$ in the original formulation.

In the equation, $u$ represents the concentration of one of the two metallic components of the alloy mixture. The parameter $\varepsilon$ is an "interaction length", which is small compared to the characteristic dimensions on the laboratory scale. The two boundary conditions in (2), the outward normal derivatives of $u$ and $\varepsilon \Delta u-\varepsilon^{-1} f(u)$ vanish on $\partial \Omega$, imply that none of the mixture can pass through the walls of the container $\Omega$; the first condition is the most natural way to ensure that the total "free energy" of the mixture decreases in time, which is required by thermodynamics, when there is no interaction between the alloy and the containing walls. The evolution of the concentration consists of two stages: the first stage (rapid in time) is known as phase separation and the second (slow in time) is known as phase coarsening. At the end of the first stage, fine-scaled phase regions are formed, which are separated by a thin region, usually considered as a hypersurface called the interface. At the end of the second stage, the solution will generically tend to a stable state, which minimizes the energy functional associated with (1). For more physical background, derivation, and discussion of the Cahn-Hilliard equation and related equations, we refer to $[12,7,11,34,35,3]$ and the references therein.

The existence of bistable states suggests that a nonconvex energy functional is associated with the equation (see the discussion below). In order to achieve broader applicability, in this paper we shall consider more general (smooth) potentials which satisfy some structural assumptions (see Section 2), and our analysis will be carried out based on these assumptions. 
We like to remark that nonsmooth potentials have also been considered in the literature for the Cahn-Hilliard equation, for that we refer to $[23,19,5,6]$ and the references therein.

It is well-known that the Cahn-Hilliard equation (1) is the $H^{-1}$-gradient flow of the functional

$$
\mathcal{J}_{\varepsilon}(u):=\int_{\Omega} \phi_{\varepsilon}(u, \nabla u) \mathrm{d} x \quad \text { and } \quad \phi_{\varepsilon}(u, \nabla u)=\frac{\varepsilon}{2}|\nabla u|^{2}+\frac{1}{\varepsilon} F(u) .
$$

Note that the energy density $\phi_{\varepsilon}(u, \nabla u)$ is a nonconvex function. It is also well-known that the $L^{2}$-gradient flow of the same energy functional $\mathcal{J}_{\varepsilon}$ is the Allen-Cahn (Ginzburg-Landau) equation

$$
u_{t}-\varepsilon \Delta u+\frac{1}{\varepsilon} f(u)=0,
$$

which was originally introduced by Allen and Cahn [3] to describe the motion of antiphase boundaries in crystalline solids (see $[12,35,28]$ and references therein). It is easy to check that the Cahn-Hilliard problem (1)-(3) conserves the total mass because its solution satisfies $\frac{d}{d t} \int_{\Omega} u(x, t) \mathrm{d} x=0$, however, the corresponding Allen-Cahn problem does not have this mass conservation property.

In addition to the reason that the Cahn-Hilliard equation is a good model to describe the phase separation and coarsening phenomena in a melted alloy, it has been extensively studied in the past decade due to its connection to an interesting and complicated free boundary problem which is known as the Mullins-Sekerka problem arising from studying solidification/melting of materials of zero specific heat, which is also known as the (two-phase) HeleShaw problem arising from the study of the pressure of immiscible fluids in the air $[36,1,16,13,11,33,32]$. It was first formally shown by Pego [36] that, as $\varepsilon \searrow 0$, the function $w:=-\varepsilon \Delta u+\varepsilon^{-1} f(u)$, known as the chemical potential, tends to a limit, which, together with a free boundary $\Gamma:=$ $\cup_{0 \leq t \leq T}\left(\Gamma_{t} \times\{t\}\right)$, satisfies the following Hele-Shaw (Mullins-Sekerka) problem:

$$
\begin{array}{ll}
\Delta w=0 & \text { in } \Omega \backslash \Gamma_{t}, t \in[0, T], \\
\frac{\partial w}{\partial n}=0 & \text { on } \partial \Omega, t \in[0, T],
\end{array}
$$

$$
\begin{aligned}
w & =\sigma \kappa & & \text { on } \Gamma_{t}, t \in[0, T], \\
V & =\frac{1}{2}\left[\frac{\partial w}{\partial n}\right]_{\Gamma_{t}} & & \text { on } \Gamma_{t}, t \in[0, T], \\
\Gamma_{0} & =\Gamma_{00} & & \text { when } t=0 .
\end{aligned}
$$


Here

$$
\sigma=\int_{-1}^{1} \sqrt{\frac{F(s)}{2}} \mathrm{~d} s .
$$

$\kappa$ and $V$ are, respectively, the mean curvature and the normal velocity of the interface $\Gamma_{t}, n$ is the unit outward normal to either $\partial \Omega$ or $\Gamma_{t},\left[\frac{\partial w}{\partial n}\right]_{\Gamma_{t}}:=$ $\frac{\partial w^{+}}{\partial n}-\frac{\partial w^{-}}{\partial n}$, and $w^{+}$and $w^{-}$are respectively the restriction of $w$ in $\Omega_{t}^{+}$and $\Omega_{t}^{-}$, the exterior and interior of $\Gamma_{t}$ in $\Omega$. Also $u \rightarrow \pm 1$ in $\Omega_{t}^{ \pm}$for all $t \in[0, T]$, as $\varepsilon \searrow 0$. The rigorous justification of this limit was successfully carried out by Stoth [39] for the radially symmetric case, and by Alikakos, Bates and Chen [1] for the general case, under the assumption that the above HeleShaw (Mullins-Sekerka) problem has a classical solution. Later, Chen [16] formulated a weak solution to the Hele-Shaw (Mullins-Sekerka) problem and showed, using an energy method, that the solution of (1)-(3) approaches, as $\varepsilon \searrow 0$, to a weak solution of the Hele-Shaw (Mullins-Sekerka) problem.

Due to the existence of nonlinearity, numerical approximations of the Cahn-Hilliard problem become a crucial mean for understanding the phase transition of the isothermal binary alloy. In addition, the connection between the Cahn-Hilliard problem and the Hele-Shaw (Mullins-Sekerka) problem leads to an attractive (indirect) approach for studying and approximating the latter through the former by taking advantage of the fact that the solution of the Cahn-Hilliard equation exists for all times [26]. The primary numerical challenge for solving the Cahn-Hilliard equation results from the presence of the parameter $\varepsilon$ in the equation, which usually is small in phase transition applications and has to be sent to zero in order to approximate the Hele-Shaw (Mullins-Sekerka) problem. As will be seen below, an appropriate numerical resolution of the solution to (1)-(3) requires proper relation of numerical scales, that is, the (spatial) mesh size $h$ and the (time) step size $k$ have to properly relate to the "interaction length" $\varepsilon$.

In the past fifteen years, different numerical approximations of the CahnHilliard equation with a fixed $\varepsilon$ have been developed and analyzed [26, 23, 25, $10,18,24,21,19,31,17]$; see also [4-6] and the references therein for finite element approximations of (1)-(3) for multi-component alloys with constant or degenerate mobility. We like to emphasize that the results of all above cited papers were established for the Cahn-Hilliard equation with a fixed "interaction length" $\varepsilon>0$. No special effort and attention were given to address issues such as how the mesh sizes $h$ and $k$ depend on $\varepsilon$ and how the error bounds depend on $\varepsilon$. In fact, since all those error estimates were derived using a Gronwall inequality type argument at the end of the derivations, it is not hard to check that all error bounds contain a factor $\exp \left(\frac{T}{\varepsilon}\right)$, which clearly is not useful when $\varepsilon$ is very small.

In contrast, our goal is to establish error bounds which depend on $\varepsilon^{-1}$ only in low polynomial order for a mixed finite element discretization of (1)-(3) 
(see below) under some reasonable constraints on spatial and temporal mesh sizes $h$ and $k$. In this paper, we focus on deriving such error estimates for the proposed mixed finite element method under minimum regularity assumptions on the initial value $u_{0}$ and on the domain $\Omega$. In a companion paper [29], we prove convergence of the solution of the fully discrete finite element method to the solution of the Hele-Shaw (Mullins-Sekerka) problem as $\varepsilon \rightarrow 0$ using the ideas and techniques developed in this paper; the program in [29] is carried out by establishing some stronger error estimates, in particular, the $L^{\infty}\left(\Omega_{T}\right)$ estimate, which only hold for more regular solutions to (1)-(3).

Our fully discrete scheme, based on a mixed variational formulation for $u$ and the chemical potential $w:=-\varepsilon \Delta u+\varepsilon^{-1} f(u)$, is defined as

$$
\begin{aligned}
\left(d_{t} U^{m}, \eta_{h}\right)+\left(\nabla W^{m}, \nabla \eta_{h}\right)=0 & \forall \eta_{h} \in \mathcal{S}_{h}, \\
\varepsilon\left(\nabla U^{m}, \nabla v_{h}\right)+\frac{1}{\varepsilon}\left(f\left(U^{m}\right)-W^{m}, v_{h}\right)=0 & \forall v_{h} \in \mathcal{S}_{h},
\end{aligned}
$$

with some starting value $U^{0} \in \mathcal{S}_{h}$. Here, $\mathcal{S}_{h} \subset H^{1}(\Omega)$ denotes the continuous piecewise linear finite element space. We consider this discrete system on the equidistant mesh $J_{k}^{1}$, and also on the stretched mesh $J_{k}^{2}:=\left\{t_{m}\right\}_{m=0}^{M}$ of local mesh sizes

$$
k_{m+1} \equiv \begin{cases}(m+1) k_{0}^{2}, & \text { for } 0 \leq t_{m+1} \leq \hat{t}_{0} \\ \gamma k_{0}, & \text { for } t_{m+1} \geq \hat{t}_{0}\end{cases}
$$

with the basic mesh size $k_{0}$, and some positive constants $\gamma, \hat{t}_{0}=O(1)$. Notice that both meshes require asymptotically the same amount of computational cost (cf. Section 3).

We now summarize our main results in this paper. Let $0<\beta<\frac{1}{2}$ be an arbitrary number. On the equidistant time mesh $J_{k}^{1}=\left\{t_{m}\right\}_{m=0}^{M}$ and for $u_{0} \in H^{2}(\Omega)$, we show a convergence rate $O\left(k^{\frac{1}{2}-\beta}\right)$ for the implicit Euler semi-discretization (see Theorem 1), which can be improved to $O\left(k_{0}^{1-\beta}\right)$ on the stretched time mesh $J_{k}^{2}=\left\{t_{m}\right\}_{m=0}^{M}$ (see Theorem 2). Theorem 3 contains error estimates, which are optimal in $h$, for the fully discrete approximation (10)-(11) on $J_{k}^{2}$. The results in Theorems 1 and 2 are obtained under general regularity assumptions for (1)-(3). Moreover, mesh constraints, which relate $\varepsilon, k_{0}$ and $h$ and under which the above convergence rates hold, are explicitly stated. The constraints indicate that small values of $\beta$ severely restrict the size of $k_{0}$.

In the case that $u_{0} \in H^{3}(\Omega)$ and either $\Omega$ is a convex polygonal domain for $N=2$ or the boundary $\partial \Omega$ is of class $C^{2,1}$ for $N=2$, 3, we show quasi-optimal order in $k$ and optimal order in $h$ convergence on the equidistant time mesh for the semi-discrete and fully discrete mixed finite element approximations, see Corollary 1 and Corollary 2. 
The analysis to be given below reveals the effects of temporal and spatial discretization independently for given initial data $u_{0} \in H^{\ell}(\Omega), \ell=2,3$. Under the premise to derive error bounds that depend only polynomially on $\frac{1}{\varepsilon}$, in order to draw conclusions about the numerical method which involves different scales $\varepsilon$ and $k_{0}, h$, the complexity of initial data (captured in terms of the parameters $\sigma_{i}$, for $\left.i=1, . .4\right)$, growth $(p>2)$ and degree of the nonmonotonicity $(\delta>0)$ of $f$, and the value $\varepsilon>0$ are all taken into account here. Our analysis is carried out for a general class of admissible double equal well potentials and initial data $u_{0} \in H^{2+\ell}, \ell=0,1$ that can be bounded in terms of negative powers of $\varepsilon$; see the general assumptions $\left(\mathrm{GA}_{1}\right)-\left(\mathrm{GA}_{3}\right)$ in Sections 2 and 3.

To establish the above error estimates, the following three ingredients play a crucial role.

(i) To establish stability estimates for the discrete solutions of the semidiscrete (in time) and the fully discrete schemes.

(ii) To handle the (nonlinear) potential term in the error equation using a spectrum estimate result due to Alikakos and Fusco [2], de Mottoni and Schatzman [20], and Chen [15] for the linearized Cahn-Hilliard operator

$$
\mathcal{L}_{C H}:=\Delta\left(\varepsilon \Delta-\frac{1}{\varepsilon} f^{\prime}(u) I\right),
$$

where $I$ denotes the identity operator and $u$ is a solution of the CahnHilliard equation (1); see Proposition 1 for details.

(iii) To establish a discrete counterpart of above spectrum estimate.

We remark that, using a similar approach parallel studies were also carried out by the authors in [28] for the Allen-Cahn equation and the related curvature driven flows, and in [30] for the classical phase field model and the related Stefan problems. On the other hand, unlike the Allen-Cahn equation which is a gradient flow for (4) in $L^{2}$, the Cahn-Hilliard equation is a gradient flow for (4) in $H^{-1}$, which makes the analysis for the Cahn-Hilliard equation in this paper more delicate and complicated than that for the Allen-Cahn equation.

The paper is organized as follows: In Section 2, we shall state some a priori estimates for the solution of (1)-(3), special attention is given to the dependence of the solution on $\varepsilon$ in various norms. In Section 3 we consider the backward Euler semi-discrete (in time) scheme for the Cahn-Hilliard equation and establish some stability estimates for the semi-discrete solution. We prove a sub-optimal error bound, which depends on $\frac{1}{\varepsilon}$ in a low polynomial order for small $\varepsilon$ as is summarized in Theorems 1-2. The spectrum estimate plays a crucial role in the proof. In Section 4, we propose a fully discrete approximation obtained by discretizing the semi-discrete scheme of Section 
3 in space using the lowest order Ciarlet-Raviart mixed finite element method. Optimal order error bounds, depending on $\frac{1}{\varepsilon}$ in a low polynomial order, are shown for the fully discrete method in Theorem 3 .

This paper is a condensed version of [27], where one can found more details and helpful discussions which could not be included here due to page limitation.

\section{Energy estimates for the differential problem}

In this section, we derive some energy estimates in various function spaces in terms of negative powers of $\varepsilon$ for the solution $u$ the Cahn-Hilliard equation (1) for given $u_{0} \in H^{2+\ell}(\Omega), \ell=0,1$. Here $J=(0, T)$, and $H^{k}(\Omega)$ denotes the standard Sobolev space of the functions which and their up to $k$ th order derivatives are $L^{2}$-integrable. Throughout this paper, the standard space, norm and inner product notation are adopted. Their definitions can be found in [10, 18]. In particular, $(\cdot, \cdot)$ denotes the standard inner product on $L^{2}(\Omega)$. Also, $c, \tilde{c}_{j}, C, \tilde{C}, \tilde{C}_{j}$ are generic positive constants which are independent of $\varepsilon$ and the time and space mesh sizes $k, k_{0}$ and $h$.

In addition, define for $r \geq 0$

$$
H^{-r}(\Omega):=\left(H^{r}(\Omega)\right)^{*}, \quad H_{0}^{-r}(\Omega):=\left\{w \in H^{-r}(\Omega) ;<w, 1>_{r}=0\right\},
$$

where $<\cdot, \cdot>_{r}$ stands for the dual product between $H^{r}(\Omega)$ and $H^{-r}(\Omega)$; we denote $L_{0}^{2}(\Omega) \equiv H_{0}^{0}(\Omega)$. For $v \in L_{0}^{2}(\Omega)$, let $v_{1}:=-\Delta^{-1} v \in H^{1}(\Omega) \cap L_{0}^{2}(\Omega)$ be the solution to

$$
-\Delta v_{1}=v \quad \text { in } \Omega, \quad \frac{\partial v_{1}}{\partial n}=0 \quad \text { on } \partial \Omega,
$$

and define $\Delta^{-\frac{1}{2}} v$ as

$$
\Delta^{-\frac{1}{2}} v:=\nabla v_{1}=-\nabla \Delta^{-1} v
$$

We make the following general assumptions on the derivative $f$ of the potential function $F$ :

\section{General Assumption $1\left(\mathbf{G A}_{1}\right)$}

1) $f=F^{\prime}$, for $F \in C^{4}(\mathbf{R})$, such that $F( \pm 1)=0$, and $F>0$ elsewhere.

2) $f^{\prime}(a)$ satisfies for some finite $2<p \leq \frac{2(N-1)}{N-2}$ and positive numbers $\tilde{c}_{i}>0, i=0, . ., 3$,

$$
\tilde{c}_{1}|a|^{p-2}-\tilde{c}_{0} \leq f^{\prime}(a) \leq \tilde{c}_{2}|a|^{p-2}+\tilde{c}_{3} .
$$


3) There exist $0<\gamma_{1} \leq 1, \gamma_{2}>0$ and $\delta>0$ such that for all $|a| \leq 2$

$$
(f(a)-f(b), a-b) \geq \gamma_{1}\left(f^{\prime}(a)(a-b), a-b\right)-\gamma_{2}|a-b|^{2+\delta} .
$$

Remark It is trivial to check that $\left(\mathrm{GA}_{1}\right)_{2}$ implies

$$
-\left(f^{\prime}(u) v, v\right) \leq \tilde{c}_{0}\|v\|_{L^{2}}^{2}, \quad \forall v \in L^{2}(\Omega),
$$

which will be utilized several times in the paper.

Example. The potential function $F(u)=\frac{1}{4}\left(u^{2}-1\right)^{2}$, consequently, $f(u)=$ $u^{3}-u$, is often used in physical and geometrical applications $[3,12,7,16]$. First, $\left(\mathrm{GA}_{1}\right)_{1}$ holds trivially. Since $f^{\prime}(u)=3 u^{2}-1,\left(\mathrm{GA}_{1}\right)_{2}$ holds with $\tilde{c}_{1}=\tilde{c}_{2}=3$ and $\tilde{c}_{0}=\tilde{c}_{3}=1$. A direct calculation gives

$$
f(a)-f(b)=(a-b)\left[f^{\prime}(a)+(a-b)^{2}-3(a-b) a\right] .
$$

Hence, $\left(\mathrm{GA}_{1}\right)_{3}$ holds with $\gamma_{1}=1, \gamma_{2}=3$, and $\delta=1$. Also, (15) holds with $\tilde{c}_{0}=1$.

In order to trace dependence of the solution on the small parameter $\varepsilon>0$, we assume that the initial function $u_{0}$ satisfies the following conditions:

\section{General Assumption $2\left(\mathbf{G A}_{2}\right)$}

Suppose that there exist positive $\varepsilon$-independent constants $m_{0}$ and $\sigma_{j}, j=$ $1,2,3$ such that

$$
\begin{aligned}
m_{0} & :=\frac{1}{|\Omega|} \int_{\Omega} u_{0}(x) \mathrm{d} x \in(-1,1), \\
\mathcal{J}_{\varepsilon}\left(u_{0}\right) & :=\frac{\varepsilon}{2}\left\|\nabla u_{0}\right\|_{L^{2}}^{2}+\frac{1}{\varepsilon}\left\|F\left(u_{0}\right)\right\|_{L^{1}} \leq C \varepsilon^{-2 \sigma_{1}}, \\
\left\|w_{0}^{\varepsilon}\right\|_{H^{\ell}} & :=\left\|-\varepsilon \Delta u_{0}^{\varepsilon}+\frac{1}{\varepsilon} f\left(u_{0}^{\varepsilon}\right)\right\|_{H^{\ell}} \leq C \varepsilon^{-\sigma_{2+\ell}}, \quad \ell=0,1 .
\end{aligned}
$$

Recall that the mixed formulation of (1)-(3) is given by

$$
\begin{aligned}
u_{t} & =\Delta w & & \text { in } \Omega_{T}, \\
w & =\frac{1}{\varepsilon} f(u)-\varepsilon \Delta u & & \text { in } \Omega_{T}, \\
\frac{\partial u}{\partial v} & =\frac{\partial w}{\partial v}=0 & & \text { on } \partial \Omega_{T}, \\
u(x, 0) & =u_{0}(x) & & \forall x \in \Omega .
\end{aligned}
$$

We refer to [26,9] and references therein for more discussions on well-posedness and regularities of the Cahn-Hilliard and the biharmonic problems.

We now state some a priori estimates for the solution of (20)-(23), which can be derived by standard test function techniques. We refer to [27] for their detailed proofs. 
Lemma 1 Suppose that $f$ satisfies $\left(G A_{1}\right)$, and $u_{0} \in H^{2}(\Omega)$ satisfies (17)(18) in $\left(G A_{2}\right)$. Then, the solution $(u, w)$ of (20)-(23) satisfies

$$
\frac{1}{|\Omega|} \int_{\Omega} u \mathrm{~d} x=m_{0} \in(-1,1), \quad \forall t \geq 0,
$$

(ii)

$$
\begin{aligned}
& \text { ess } \sup _{[0, \infty]}\left\{\frac{\varepsilon}{2}\|\nabla u\|_{L^{2}}^{2}+\frac{1}{\varepsilon}\|F(u)\|_{L^{1}}\right\} \\
& +\left\{\begin{array}{c}
\int_{0}^{\infty}\left\|u_{t}(s)\right\|_{H^{-1}}^{2} \mathrm{~d} s \\
\int_{0}^{\infty}\|\nabla w(s)\|_{L^{2}}^{2} \mathrm{~d} s
\end{array}\right\}=\mathcal{J}_{\varepsilon}\left(u_{0}\right),
\end{aligned}
$$

(iii)

$$
\int_{0}^{\infty}\|\Delta u(s)\|_{L^{2}}^{2} \mathrm{~d} s \leq C \varepsilon^{-\left(2 \sigma_{1}+3\right)}
$$

(iv) $\quad$ ess $\sup _{[0, \infty]}\left\|\Delta^{-1} u_{t}\right\|_{L^{2}}^{2}+\left\{\begin{array}{l}\varepsilon \int_{0}^{\infty}\left\|u_{t}(s)\right\|_{L^{2}}^{2} \mathrm{~d} s \\ \varepsilon \int_{0}^{\infty}\|\Delta w(s)\|_{L^{2}}^{2} \mathrm{~d} s\end{array}\right\}$

$$
\leq C \varepsilon^{-\max \left\{2 \sigma_{1}(p-1)+p+1,2 \sigma_{2}\right\}},
$$

(v) $\quad$ ess $\sup \|\Delta u\|_{L^{2}}^{2} \leq C \rho_{1}(\varepsilon)$,

$$
[0, \infty]
$$

(vi) $\quad$ ess $\sup _{[0, \infty]} \tau(t)\left\|u_{t}\right\|_{H^{-1}}^{2}+\varepsilon \int_{0}^{\infty} \tau(s)\left\|\nabla u_{t}\right\|_{L^{2}}^{2} \mathrm{~d} s \leq C \varepsilon^{-\left(2 \sigma_{1}+3\right)}$,

(vii) $\quad$ ess $\sup _{[0, \infty]} \tau(t)\left\|u_{t}\right\|_{L^{2}}^{2}+\varepsilon \int_{0}^{\infty} \tau(s)\left\|\Delta u_{t}\right\|_{L^{2}}^{2} \mathrm{~d} s$

$$
\leq C \varepsilon^{-\max \left\{2 \sigma_{1}(p-1)+p+4,2 \sigma_{2}+1\right\}},
$$

$$
\int_{0}^{\infty}\left\|\Delta^{-2} u_{t t}(s)\right\|_{L^{2}}^{2} \mathrm{~d} s \leq C \rho_{2}(\varepsilon),
$$

$$
\int_{0}^{\infty} \tau(s)\left\|\Delta^{-1} u_{t t}(s)\right\|_{H^{-1}}^{2} \mathrm{~d} s \leq C \rho_{2}(\varepsilon),
$$

where

$$
\begin{aligned}
& \rho_{1}(\varepsilon):=\varepsilon^{-\max \left\{2 \sigma_{1}(p-1)+p+3,2 \sigma_{1}+3,2\left(\sigma_{2}+1\right)\right\}}, \\
& \rho_{2}(\varepsilon):=\varepsilon^{-\max \left\{2\left(\sigma_{1}(2 p-3)+p+1\right), 2 \sigma_{2}+1+2 \sigma_{1}(p-2)+p\right\}},
\end{aligned}
$$

and $\tau \equiv \tau(t)=\min \left\{t, \hat{t}_{0}\right\}$, for any fixed small number $0<\hat{t}_{0}=O(1)$.

The above estimates are derived under the minimum regularity assumption $u_{0} \in H^{2}(\Omega)$. They show the strong dependency of the solution on negative powers of $\varepsilon$ in high norms. On the other hand, we show in the following that the estimates will improve drastically if the initial data $u_{0} \in H^{3}(\Omega)$ and the boundary $\partial \Omega \in C^{2,1}$ are considered. Alternatively, the subsequent results also hold for convex polygonal domains in the case $N=2$; see [27] for a short proof. 
Lemma 2 Suppose that $f$ satisfies $\left(G A_{1}\right)$, and $u_{0} \in H^{3}(\Omega)$ satisfies $\left(G A_{2}\right)$, and $\partial \Omega$ is of class $C^{2,1}$. Then the solution of (20)-(23) satisfies the following estimates:

$$
\frac{1}{|\Omega|} \int_{\Omega} u \mathrm{~d} x=m_{0} \in(-1,1), \quad \forall t \geq 0,
$$

$$
\text { ess } \sup _{[0, \infty]}\left\|u_{t}\right\|_{H^{-1}}^{2}+\varepsilon \int_{0}^{\infty}\left\|\nabla u_{t}\right\|_{L^{2}}^{2} \mathrm{~d} s \leq C \varepsilon^{-\max \left\{2 \sigma_{1}+3,2 \sigma_{3}\right\}} \text {, }
$$

$$
\begin{aligned}
& \text { ess } \sup _{[0, \infty]}\|\nabla u\|_{L^{2}}^{2}+\varepsilon \int_{0}^{\infty}\|u(s)\|_{H^{3}}^{2} \mathrm{~d} s \leq C \varepsilon^{-\left\{2 \sigma_{1}(p-1)+p+4\right\}}, \\
& \int_{0}^{\infty}\left\|\Delta^{-1} u_{t t}\right\|_{H^{-1}}^{2} \mathrm{~d} s \leq C \tilde{\rho}_{2}(\varepsilon)
\end{aligned}
$$

where

$$
\tilde{\rho}_{2}(\varepsilon):=\varepsilon^{-\max \left\{2 \sigma_{1}(2 p-3)+2 p+2,2 \sigma_{1}(p-2)+2 \sigma_{2}+p+1,2 \sigma_{3}-1\right\}} .
$$

We conclude this section by citing the following result of $[2,15]$ on a low bound estimate of the spectrum of the linearized Cahn-Hilliard operator $\mathcal{L}_{C H}$ in (13). This result plays a crucial role in our error analysis; it describes the coarsening dynamics and applies to cases where (i) $u$ has a special profile approximating a smooth hypersurface as detailed on page 1374 of [15] (see also [2]), and (ii) the related problem (5)-(9) has a smooth solution. Both assumptions are assumed to hold throughout the remainder of the paper.

Proposition 1 Suppose that $\left(G A_{1}\right)$ holds. Then there exist $0<\varepsilon_{0}<<1$ and another positive constant $C_{0}$ such that the principle eigenvalue of the linearized Cahn-Hilliard operator $\mathcal{L}_{C H}$ in (13) satisfies for all $t \in[0, T]$

$$
\lambda_{C H} \equiv \inf _{\substack{0 \neq \psi \in H^{1}(\Omega) \\ \Delta w=\psi}} \frac{\varepsilon\|\nabla \psi\|_{L^{2}}^{2}+\frac{1}{\varepsilon}\left(f^{\prime}(u(\cdot, t)) \psi, \psi\right)}{\|\nabla w\|_{L^{2}}^{2}} \geq-C_{0}
$$

for $\varepsilon \in\left(0, \varepsilon_{0}\right)$. Where $u$ is the solution to the Cahn-Hilliard problem (1)-(3).

Proof. It was proved in [2] for $N=2$ and in [15] for $N \geq 2$ that (24) is valid provided that the function $u$ (which does not have to be the solution to the Cahn-Hilliard equation) satisfies some special profile (cf. page 638 of [2] and page 1374 of [15]). It was shown in [1] that the solution to the CahnHilliard problem (1)-(3) indeed satisfies the required profile (cf. Theorems 4.12 and 2.1 of [1]) for sufficiently small $\varepsilon$; the statement of the subsequent proposition then follows from combining these two results. 


\section{Error analysis for a semi-discrete (in time) approximation}

Consider a semi-discrete approximation of (20)-(23) on mesh $J_{k}^{1}:=\left\{t_{m}\right\}_{m=0}^{M}$ : Find $\left\{\left(u^{m}, w^{m}\right)\right\}_{m=1}^{M} \in\left[H^{1}(\Omega)\right]^{2}$ such that for every $0 \leq m \leq M$

$$
\begin{aligned}
& \left(d_{t} u^{m+1}, \eta\right)+\left(\nabla w^{m+1}, \nabla \eta\right)=0 \quad \forall \eta \in H^{1}(\Omega), \\
& \varepsilon\left(\nabla u^{m+1}, \nabla v\right)+\frac{1}{\varepsilon}\left(f\left(u^{m+1}\right), v\right)=\left(w^{m+1}, v\right) \quad \forall v \in H^{1}(\Omega),
\end{aligned}
$$

with $u^{0}=u_{0}$. Here $J_{k}^{1}:=\left\{t_{m}\right\}_{m=0}^{M}$ is a quasi-uniform partition of $[0, T]$ of mesh size $k:=\frac{T}{M}$. Also, $d_{t} u^{m+1}:=\left(u^{m+1}-u^{m}\right) / k$.

It turns out from the subsequent analysis that this scheme on the time mesh $J_{k}^{1}$ only performs sub-optimal (see Theorem 1) for general regularities (see Lemma 1) and quasi-optimal (see Corollary 1) under additional assumptions on regularity of the problem (see Lemma 2). The reason for the sub-optimal convergence in the case of general regularities is the lack of an estimate for $\Delta^{-1} u_{t t}$ in $L^{2}\left(J, H^{-1}(\Omega)\right)$.

In order to construct an optimally convergent time discretization scheme for (20)-(21) in the case $u_{0} \in H^{2}(\Omega)$, we suggest to compute iterates $u^{m+1}$ of (25)-(26) on a stretched mesh $J_{k}^{2}:=\left\{t_{m}\right\}_{m=0}^{M}$ introduced in (12), for a basic mesh size $k_{0}$ and $\hat{t}_{0}=O(1)$; see (Chapter 10 of [37]). Obviously, this grid structure is very fine near the origin, with increasing mesh size at increasing times, and requires $O\left(k_{0}^{-1}\right)$ iteration steps to overcome the critical time interval $\left[0, \hat{t}_{0}\right]$. It will be proved in Theorem 2 that the benefit of using the stretched mesh $J_{k}^{2}$ is that it results in quasi-optimal error bounds.

Finally, we need a stability property for scheme (25)-(26) with respect to admissible discretizations of the nonlinear term.

\section{General Assumption $3\left(\mathbf{G A}_{3}\right)$}

Suppose that there exists $\alpha_{0} \geq 0,0<\gamma_{3}<1$, and $\tilde{c}_{4}>0$ such that $f$ satisfies for any $0<k_{m} \leq \varepsilon^{\alpha_{0}}$ and any set of discrete (in time) functions $\left\{\phi^{m}\right\}_{m=0}^{M} \in H^{1}(\Omega)$

$$
\begin{gathered}
\gamma_{3} \sum_{m=1}^{\ell} k_{m}\left(\left\|d_{t} \phi^{m}\right\|_{H^{-1}}^{2}+k_{m} \varepsilon\left\|\nabla d_{t} \phi^{m}\right\|_{L^{2}}^{2}\right) \\
+\frac{1}{\varepsilon} \sum_{m=1}^{\ell} k_{m}\left(f\left(\phi^{m}\right), d_{t} \phi^{m}\right)+\tilde{c}_{4} \mathcal{J}_{\varepsilon}\left(\phi^{0}\right) \\
\geq \frac{\tilde{c}_{4}}{\varepsilon}\left\|F\left(\phi^{\ell}\right)\right\|_{L^{1}} \quad \forall \ell \leq M .
\end{gathered}
$$


Note that this assumption holds for $f(u)=u^{3}-u$, we refer to $[28,27]$ for its proof.

A direct consequence of (27) are the following stability estimates for the scheme (25)-(26) which hold on both meshes $J_{k}^{1}$ and $J_{k}^{2}$. Moreover, additional estimates in strong norms are shown for the stretched mesh, which indicates its stabilizing effect.

Lemma 3 For $k_{m} \leq \varepsilon^{\alpha_{0}}$ and $u_{0} \in H^{2}(\Omega)$, the solution of the scheme (25)(26) satisfies the following estimates on both meshes $J_{k}^{1}$ and $J_{k}^{2}$.

(i) $\frac{1}{|\Omega|} \int_{\Omega} u^{m} \mathrm{~d} x=m_{0} \in(-1,1), \quad \forall m \geq 0$,

(ii) $\max _{0 \leq m \leq M}\left\{\varepsilon\left\|\nabla u^{m}\right\|_{L^{2}}^{2}+\frac{1}{\varepsilon}\left\|F\left(u^{m}\right)\right\|_{L^{1}}\right\}$

$$
\begin{aligned}
& +\sum_{m=1}^{M} k_{m}\left\{\left\|\nabla w^{m}\right\|_{L^{2}}^{2}+\left\|d_{t} u^{m}\right\|_{H^{-1}}^{2}+\varepsilon k_{m}\left\|\nabla d_{t} u^{m}\right\|_{L^{2}}^{2}\right\} \\
& \leq 2 \tilde{c}_{4} \mathcal{J}_{\varepsilon}\left(u^{0}\right), \\
& \text { (iii) } \quad \sum_{m=0}^{M} k_{m}\left\|\Delta u^{m}\right\|_{L^{2}}^{2} \leq C \varepsilon^{-\left(2 \sigma_{1}+3\right)} .
\end{aligned}
$$

Moreover, there hold for $J_{k}^{1}$

$$
\begin{aligned}
& \text { (iv) } \max _{1 \leq m \leq M}\left\|\Delta^{-1} d_{t} u^{m}\right\|_{L^{2}}^{2}+\varepsilon k \sum_{m=1}^{M}\left\|d_{t} u^{m}\right\|_{L^{2}}^{2} \\
& \leq C \varepsilon^{-\max \left\{2 \sigma_{1}(p-1)+p+1,2 \sigma_{2}\right\}}, \\
& \text { (v) } \max _{0 \leq m \leq M}\left\|\Delta u^{m}\right\|_{L^{2}}^{2} \leq C \rho_{1}(\varepsilon),
\end{aligned}
$$

and for $J_{k}^{2}$

$$
\begin{aligned}
& \text { (vi) } \max _{1 \leq m \leq M}\left\{\left\|\tilde{d}_{t} u^{m}\right\|_{H^{-1}}^{2}+\varepsilon\left\|\nabla u^{m}\right\|_{L^{2}}^{2}\right\}+\frac{k_{0}^{2}}{2} \sum_{m=1}^{M}\left\{\left\|\tilde{d}_{t}^{2} u^{m}\right\|_{H^{-1}}^{2}\right. \\
& \left.+\frac{\varepsilon k_{0}^{2}}{2}\left\|\nabla \tilde{d}_{t} u^{m}\right\|_{L^{2}}^{2}\right\}+\frac{\varepsilon}{2} \sum_{m=1}^{M} k_{m}\left\|\nabla \tilde{d}_{t} u^{m}\right\|_{L^{2}}^{2} \\
& \leq C\left\{\varepsilon^{-\left(2 \sigma_{1}+3\right)}+\ln \left(\frac{1}{k_{0}}\right) \varepsilon^{-\left\{2 \sigma_{1}(p-1)+p+\frac{3}{2}\right\}}\right\},
\end{aligned}
$$




$$
\begin{aligned}
& \text { (vii) } \max _{1 \leq m \leq M}\left\{\left\|\tilde{d}_{t} u^{m}\right\|_{L^{2}}^{2}+\varepsilon\left\|\Delta u^{m}\right\|_{L^{2}}^{2}\right\}+\frac{k_{0}^{2}}{2} \sum_{m=1}^{M}\left\{\left\|\tilde{d}_{t}^{2} u^{m}\right\|_{L^{2}}^{2}\right. \\
& \left.+\varepsilon\left\|\Delta \tilde{d}_{t} u^{m}\right\|_{L^{2}}^{2}\right\} \\
& +\frac{\varepsilon}{2} \sum_{m=1}^{M} k_{m}\left\|\Delta \tilde{d}_{t} u^{m}\right\|_{L^{2}}^{2} \leq C \varepsilon \tilde{\rho}_{1}(\varepsilon)
\end{aligned}
$$

where $\tilde{d}_{t} \varphi^{m+1}:=\frac{1}{k_{0}}\left\{\varphi^{m+1}-\varphi^{m}\right\}$, and

$$
\begin{aligned}
\tilde{\rho}_{1}(\varepsilon)= & \max \left\{\operatorname { l n } ( \frac { 1 } { k _ { 0 } } ) \varepsilon ^ { - ( ( 2 \sigma _ { 1 } + 1 ) ( p - 2 ) + 4 ) } \left\{\varepsilon^{-\left(2 \sigma_{1}+3\right)}\right.\right. \\
& \left.+\ln \left(\frac{1}{k_{0}}\right) \varepsilon^{-\left(2 \sigma_{1}(p-1)+p+\frac{3}{2}\right)}\right\} \\
& \left.+C\left\{\varepsilon^{-2\left(\left(\sigma_{1}+\sigma_{2}\right)+1\right)}+\varepsilon^{-\left(2 \sigma_{1}(p-2)+2 \sigma_{1}+p+1\right)}\right\}, \rho_{1}(\varepsilon)\right\} .
\end{aligned}
$$

In addition, under the assumptions of Lemma 2, there also holds for the mesh $J_{k}^{1}$

$$
\begin{aligned}
& \text { (viii) } \max _{1 \leq m \leq M}\left\|d_{t} u^{m}\right\|_{H^{-1}}^{2}+\varepsilon k \sum_{m=1}^{M}\left\|\nabla d_{t} u^{m}\right\|_{L^{2}}^{2} \leq C \varepsilon^{-\max \left\{2 \sigma_{1}+3,2 \sigma_{3}\right\}}, \\
& \text { (ix) } \max _{0 \leq m \leq M}\left\|\nabla u^{m}\right\|_{L^{2}}^{2}+\varepsilon k \sum_{m=0}^{M}\left\|u^{m}\right\|_{H^{3}}^{2} \leq C \varepsilon^{-\left\{2 \sigma_{1}(p-1)+p+4\right\}} .
\end{aligned}
$$

Remark Assumption $\left(\mathrm{GA}_{3}\right)$ is a stability requirement for a "good" time discretization, which holds under mesh constraint $k_{m} \leq \varepsilon^{\alpha_{0}}$. In the literature, modifications of (25)-(26) have been proposed to relax this restriction. One strategy is to decompose $F$ into $F^{+}-F^{-}$, where $F^{ \pm}$are two convex functions, and to treat the first term implicitly and the second explicitly (cf. [8]). Another strategy is to use a certain combination of $f$ evaluated at different time steps (cf. [28]). Despite the advantage of having a discrete energy law without parameter restrictions, we prefer the scheme (25)-(26) for its simpler structure and simpler subsequent error analysis.

Proof. For verifications of the estimates (i)-(v), (viii), (ix), see [27]. Here, we only give proofs of (vi), (vii) on the time-mesh $J_{k}^{2}$.

(vi) We apply $\tilde{d}_{t}$ to this equation and find

$$
\tilde{d}_{t}^{2} u^{m+1}-m k_{0} \Delta \tilde{d}_{t} w^{m+1}-\Delta w^{m+1}=0 .
$$


We test the above equation with $-\Delta^{-1} \tilde{d}_{t} u^{m+1}$ and find

$$
\begin{aligned}
& \frac{1}{2} \tilde{d}_{t}\left\|\Delta^{-\frac{1}{2}} \tilde{d}_{t} u^{m+1}\right\|_{L^{2}}^{2}+\frac{k_{0}}{2}\left\|\Delta^{-\frac{1}{2}} \tilde{d}_{t}^{2} u^{m+1}\right\|_{L^{2}}^{2} \\
& +\varepsilon m k_{0}\left\|\nabla \tilde{d}_{t} u^{m+1}\right\|_{L^{2}}^{2} \\
& +\frac{\varepsilon}{2} \tilde{d}_{t}\left\|\nabla u^{m+1}\right\|_{L^{2}}^{2}+\frac{\varepsilon k_{0}}{2}\left\|\nabla \tilde{d}_{t} u^{m+1}\right\|_{L^{2}}^{2} \\
& \quad=-\frac{m k_{0}}{\varepsilon}\left(f^{\prime}(\xi),\left|\tilde{d}_{t} u^{m+1}\right|^{2}\right) \\
& +\frac{1}{\varepsilon}\left(f^{\prime}\left(u^{m+1}\right) \nabla u^{m+1}, \nabla \Delta^{-1} \tilde{d}_{t} u^{m+1}\right) .
\end{aligned}
$$

Here, $\xi$ is a value between $u^{m}$ and $u^{m+1}$. Using the upper bound

$$
\left\|f^{\prime}\left(u^{m}\right)\right\|_{L^{3}}^{2}\left\|\Delta^{-1 / 2} \tilde{d}_{t} u^{m}\right\|_{L^{2}}^{2} \leq\left(\tilde{c}_{2}\left\|u^{m}\right\|_{L^{3(p-2)}}^{2(p-2)}+\tilde{c}_{3}\right)\left\|\Delta^{-1 / 2} \tilde{d}_{t} u^{m}\right\|_{L^{6}}^{2},
$$

the last line can be bounded by

$$
\begin{aligned}
& \frac{\tilde{c}_{0} m k_{0}}{\varepsilon}\left\|\tilde{d}_{t} u^{m+1}\right\|_{L^{2}}^{2}+\frac{C}{\varepsilon}\left\|f^{\prime}\left(u^{m+1}\right)\right\|_{L^{3}}\left\|\nabla u^{m+1}\right\|_{L^{2}}\left\|\nabla \Delta^{-1} \tilde{d}_{t} u^{m+1}\right\|_{L^{6}} \\
& \leq \frac{\varepsilon m k_{0}}{4}\left\|\nabla \tilde{d}_{t} u^{m+1}\right\|_{L^{2}}^{2}+\frac{C m k_{0}}{\varepsilon^{3}}\left\|\nabla \Delta^{-1} \tilde{d}_{t} u^{m+1}\right\|_{L^{2}}^{2} \\
& \quad+\frac{1}{\varepsilon}\left\|f^{\prime}\left(u^{m+1}\right)\right\|_{L^{3}}\left\|\nabla u^{m+1}\right\|_{L^{2}}\left\|\nabla \Delta^{-1} \tilde{d}_{t} u^{m+1}\right\|_{L^{6}} .
\end{aligned}
$$

Let $\delta>0$. Using (31) the last contribution is bounded by

$$
\begin{aligned}
\frac{C \delta}{\varepsilon^{5 / 2}} & \left(\tilde{c}_{2}\left\|u^{m+1}\right\|_{L^{3(p-2)}}^{2(p-2)}+\tilde{c}_{3}\right)\left\|\nabla u^{m+1}\right\|_{L^{2}}^{2}+\frac{\sqrt{\varepsilon}}{\delta}\left\|\tilde{d}_{t} u^{m+1}\right\|_{L^{2}}^{2} \\
\leq & C \delta \varepsilon^{-\left\{\left(2 \sigma_{1}+1\right)(p-2)+2 \sigma_{1}+\frac{7}{2}\right\}} \\
& +\frac{\varepsilon m k_{0}}{4}\left\|\nabla \tilde{d}_{t} u^{m+1}\right\|_{L^{2}}^{2}+\frac{C}{k_{0} m \delta^{2}}\left\|\nabla \Delta^{-1} \tilde{d}_{t} u^{m+1}\right\|_{L^{2}}^{2} .
\end{aligned}
$$

We insert this into (30) and multiply by $k_{0}$, finally sum over $m$ from 1 to $\ell$ $(\leq M)$. Note that $\left|\sum_{m=1}^{M} \frac{1}{m}-\ln \frac{1}{M}\right|<1$, and observe $\tilde{d}_{t} u^{m}=k_{0} m d_{t} u^{m}$ to find

$$
\sum_{m=1}^{M} k_{m}\left\|\Delta^{-\frac{1}{2}} \tilde{d}_{t} u^{m}\right\|_{L^{2}}^{2} \leq \sum_{m=1}^{M} k_{m}\left\|\Delta^{-\frac{1}{2}} d_{t} u^{m}\right\|_{L^{2}}^{2} .
$$


From (i) and discrete Gronwall's inequality, we find for the choice $\delta^{2}=$ $\ln \left(\frac{1}{k_{0}}\right)$,

$$
\begin{aligned}
\frac{1}{2} \| & \Delta^{-\frac{1}{2}} \tilde{d}_{t} u^{\ell}\left\|_{L^{2}}^{2}+\frac{k_{0}^{2}}{2} \sum_{m=1}^{M}\right\| \Delta^{-\frac{1}{2}} \tilde{d}_{t}^{2} u^{m}\left\|_{L^{2}}^{2}+\frac{\varepsilon}{4} \sum_{m=1}^{M} k_{m}\right\| \nabla \tilde{d}_{t} u^{m} \|_{L^{2}}^{2} \\
+ & \frac{\varepsilon}{2}\left\|\nabla u^{\ell}\right\|_{L^{2}}^{2}+\frac{\varepsilon k_{0}^{2}}{2} \sum_{m=1}^{M}\left\|\nabla \tilde{d}_{t} u^{m+1}\right\|_{L^{2}}^{2} \\
\leq & C\left\{\varepsilon^{-\left(2 \sigma_{1}+3\right)}+\ln \left(\frac{1}{k_{0}}\right) \varepsilon^{-\left\{2 \sigma_{1}(p-1)+p+\frac{3}{2}\right\}}\right\} \\
+ & \frac{1}{2}\left\{\left\|\Delta^{-\frac{1}{2}} \tilde{d}_{t} u^{1}\right\|_{L^{2}}^{2}+\varepsilon\left\|\nabla u^{1}\right\|_{L^{2}}^{2}\right\} .
\end{aligned}
$$

By (ii), the last two terms can be bounded by $\varepsilon^{-2 \sigma_{1}}$. We benefit at this point again from the scaling of the stretched mesh $J_{k}^{2}$,

$$
\left\|\Delta^{-\frac{1}{2}} \frac{u^{1}-u^{0}}{k_{0}}\right\|_{L^{2}}^{2} \leq k_{0}^{2}\left\|\Delta^{-\frac{1}{2}} d_{t} u^{1}\right\|_{L^{2}}^{2} \leq C \varepsilon^{-2 \sigma_{1}} .
$$

(vii) We test $\tilde{d}_{t} u^{m+1}-(m+1) k_{0} \Delta w^{m+1}=0$ with $\tilde{d}_{t} u^{m+1}$. In the sequel, $\xi$ is a value between $u^{m}$ and $u^{m+1}$.

$$
\begin{aligned}
& \frac{1}{2} \tilde{d}_{t}\left\|\tilde{d}_{t} u^{m+1}\right\|_{L^{2}}^{2}+\frac{k_{0}}{2}\left\|\tilde{d}_{t}^{2} u^{m+1}\right\|_{L^{2}}^{2}+\varepsilon m k_{0}\left\|\Delta \tilde{d}_{t} u^{m+1}\right\|_{L^{2}}^{2} \\
& +\frac{\varepsilon}{2} \tilde{d}_{t}\left\|\Delta u^{m+1}\right\|_{L^{2}}^{2}+\frac{\varepsilon k_{0}}{2}\left\|\Delta \tilde{d}_{t} u^{m+1}\right\|_{L^{2}}^{2} \\
& =\frac{m k_{0}}{\varepsilon}\left(f^{\prime}(\xi) \tilde{d}_{t} u^{m+1}, \Delta \tilde{d}_{t} u^{m+1}\right)-\frac{1}{\varepsilon}\left(f^{\prime}\left(u^{m+1}\right) \nabla u^{m+1}, \nabla \tilde{d}_{t} u^{m+1}\right)
\end{aligned}
$$

We multiply by $k_{0}$, sum over $m$ from 1 to $M$, use the upper bound from (31) and the estimate

$$
\begin{aligned}
& \frac{1}{\varepsilon}\left\|f^{\prime}\left(u^{m}\right)\right\|_{L^{3}}\left\|\nabla u^{m}\right\|_{L^{2}}\left\|\nabla \tilde{d}_{t} u^{m}\right\|_{L^{6}} \\
& \quad \leq \frac{C}{\varepsilon^{3} m k_{0}}\left\|f^{\prime}\left(u^{m}\right)\right\|_{L^{3}}^{2}\left\|\nabla u^{m}\right\|_{L^{2}}^{2}+\frac{\varepsilon m k_{0}}{2}\left\|\Delta \tilde{d}_{t} u^{m}\right\|_{L^{2}}^{2} \\
& \quad \leq \frac{C}{\varepsilon^{3} m k_{0}}\left(\tilde{c}_{2}\left\|u^{m}\right\|_{L^{3(p-2)}}^{2(p-2)}+\tilde{c}_{3}\right)\left\|\nabla u^{m}\right\|_{L^{2}}^{2}+\frac{\varepsilon m k_{0}}{2}\left\|\Delta \tilde{d}_{t} u^{m}\right\|_{L^{2}}^{2} \\
& \quad \leq \frac{C}{m k_{0}} \varepsilon^{-\left(2 \sigma_{1}+1\right)(p-2)-3}+\frac{\varepsilon m k_{0}}{2}\left\|\Delta \tilde{d}_{t} u^{m}\right\|_{L^{2}}^{2}
\end{aligned}
$$


to get from (33)

$$
\begin{aligned}
\max _{2 \leq m \leq M} & \frac{1}{2}\left\{\left\|\tilde{d}_{t} u^{m}\right\|_{L^{2}}^{2}+\varepsilon\left\|\Delta u^{m}\right\|_{L^{2}}^{2}\right\}+\frac{k_{0}^{2}}{2} \sum_{m=2}^{M}\left\{\left\|\tilde{d}_{t}^{2} u^{m}\right\|_{L^{2}}^{2}\right. \\
& \left.+\frac{\varepsilon}{2}\left\|\Delta \tilde{d}_{t} u^{m}\right\|_{L^{2}}^{2}\right\}+\frac{\varepsilon}{2} \sum_{m=2}^{M} k_{m}\left\|\Delta \tilde{d}_{t} u^{m}\right\|_{L^{2}}^{2} \\
\leq & \frac{1}{2}\left\{\left\|\tilde{d}_{t} u^{1}\right\|_{L^{2}}^{2}+\varepsilon\left\|\Delta u^{1}\right\|_{L^{2}}^{2}\right\}+C \varepsilon^{-\left\{2 \sigma_{1}(p-2)+p+1\right\}} \ln \left(\frac{1}{k_{0}}\right) \\
& +\frac{C}{\varepsilon^{3}} \sum_{m=2}^{M} k_{m}\left\|f^{\prime}(\xi)\right\|_{L^{3}}^{2}\left\|\tilde{d}_{t} u^{m}\right\|_{L^{6}}^{2} .
\end{aligned}
$$

The logarithmic term comes again from the bound $\left|\sum_{m=1}^{M} \frac{1}{m}-\ln \frac{1}{M}\right|<1$. The last term in (34) is estimated by

$$
\begin{gathered}
\frac{C}{\varepsilon^{3}} \sum_{m=1}^{M} k_{m}\left(\tilde{c}_{2}\left\|u^{m}\right\|_{L^{3(p-2)}}^{2(p-2)}+\tilde{c}_{3}\right)\left\|\nabla \tilde{d}_{t} u^{m}\right\|_{L^{2}}^{2} \\
\leq C \ln \left(\frac{1}{k_{0}}\right) \varepsilon^{-\left(\left(2 \sigma_{1}+1\right)(p-2)+3\right)}\left\{\varepsilon^{-\left(2 \sigma_{1}+3\right)}\right. \\
\left.\quad+\ln \left(\frac{1}{k_{0}}\right) \varepsilon^{-\left(2 \sigma_{1}(p-1)+p+\frac{3}{2}\right)}\right\} .
\end{gathered}
$$

The last inequality follows from an elementary calculation.

The first two terms on the right hand side of (34) are bounded because of the structure of $J_{k}^{2}$. Therefore, taking $m=0$ in the equation at the beginning of (vii) and testing the equation with $\tilde{d}_{t} u^{1}$ leads to

$$
\begin{aligned}
& \left\|\tilde{d}_{t} u^{1}\right\|_{L^{2}}^{2}+\frac{\varepsilon k_{0}}{2} \tilde{d}_{t}\left\|\Delta u^{1}\right\|_{L^{2}}^{2}=-\frac{k_{0}}{\varepsilon}\left(f^{\prime}\left(u^{1}\right) \nabla u^{1}, \nabla \tilde{d}_{t} u^{1}\right) \\
& \leq \frac{\tilde{c}_{0}}{\varepsilon}\left\|\nabla u^{1}\right\|_{L^{2}}^{2}+\frac{1}{\varepsilon}\left\|f^{\prime}\left(u^{1}\right)\right\|_{L^{3}}\left\|\nabla u^{1}\right\|_{L^{2}}\left\|\nabla u^{0}\right\|_{L^{6}} .
\end{aligned}
$$

Similar to (31), using $\left(\mathrm{GA}_{2}\right)$ the above inequality is continued by

$$
\begin{aligned}
& \frac{2 \tilde{c}_{0}}{\varepsilon}\left\|\nabla u^{1}\right\|_{L^{2}}^{2}+\frac{1}{4 \tilde{c}_{0} \varepsilon}\left\|f^{\prime}\left(u^{1}\right)\right\|_{L^{3}}^{2}\left\|\nabla u^{0}\right\|_{L^{6}}^{2} \\
& \quad \leq \frac{2 \tilde{c}_{0}}{\varepsilon}\left\|\nabla u^{1}\right\|_{L^{2}}^{2}+\frac{1}{4 \tilde{c}_{0} \varepsilon}\left(\left\|u^{1}\right\|_{L^{3(p-2)}}^{2(p-2)}+\tilde{c}_{3}\right)\left\|\Delta u^{0}\right\|_{L^{2}}^{2} \\
& \quad \leq C\left\{\varepsilon^{-2\left(\sigma_{1}+1\right)}+\varepsilon^{-\left(2 \sigma_{1}(p-2)+p-1\right)}\right\}\left\{\varepsilon^{-\left(2 \sigma_{1}+2\right)}+\varepsilon^{-\left(2 \sigma_{1}+1\right)}\right\} .
\end{aligned}
$$


Using (36) and (35), we find the following upper bound for (34)

$$
\begin{aligned}
C & \ln \left(\frac{1}{k_{0}}\right) \varepsilon^{-\left(\left(2 \sigma_{1}+1\right)(p-2)+3\right)}\left\{\varepsilon^{-\left(2 \sigma_{1}+3\right)}+\ln \left(\frac{1}{k_{0}}\right) \varepsilon^{-\left(2 \sigma_{1}(p-1)+p+\frac{3}{2}\right)}\right\} \\
& +C\left\{\varepsilon^{-2\left(\left(\sigma_{1}+\sigma_{2}\right)+1\right)}+\varepsilon^{-\left(2 \sigma_{1}(p-2)+2 \sigma_{1}+p+1\right)}\right\} .
\end{aligned}
$$

This concludes the proof.

\subsection{Error estimates for the scheme (25)-(26)}

In this subsection, we present the error analysis for (25)-(26) under the assumptions $\left(\mathrm{GA}_{1}\right)-\left(\mathrm{GA}_{3}\right)$, starting with the mesh $J_{k}^{1}$.

Theorem 1 Let $\left\{\left(u^{m}, w^{m}\right)\right\}_{m=0}^{M}$ solve (25)-(26) on an equidistant mesh $J_{k}^{1}=$ $\left\{t_{m}\right\}_{m=0}^{M}$ of mesh size $O(k)$, and $u_{0} \in H^{2}(\Omega)$. Suppose $\left(G A_{1}\right)-\left(G A_{3}\right)$ hold, and $1<\delta<\frac{8}{4-N}$. Let $\rho_{1}(\varepsilon)$ and $\rho_{2}(\varepsilon)$ be same as in Lemma 1, and

$$
\begin{aligned}
& \rho_{3}(\varepsilon)=\rho_{2}(\varepsilon)\left[\rho_{1}(\varepsilon)\right]^{-\frac{2 N \delta}{8-(4-N) \delta}} \varepsilon^{\frac{\left(2 \sigma_{1}+1\right)(4-N) \delta}{8-(4-N) \delta}+2\left(\sigma_{1}+2\right)}, \\
& \rho_{4}(N, \beta)=\left(1+\beta+\frac{4(4-N) \delta}{8-(4-N) \delta}\right)^{-1}, \\
& \rho_{5}(\varepsilon, N)=\left[\rho_{1}(\varepsilon)\right]^{-2 N}\left[\rho_{2}(\varepsilon)\right]^{N-4}, \\
& \rho_{6}(N, \beta)=\delta\left[(4-N) \delta-\beta(8+2(4-N) \delta]^{-1}\right. \text {. }
\end{aligned}
$$

For fixed positive values $0<\beta<\frac{1}{2}$, let $k$ satisfy the following constraint

$$
k \leq \tilde{C} \min \left\{\varepsilon^{\frac{3}{\beta}}, \varepsilon^{\alpha_{0}},\left[\rho_{3}(\varepsilon, N)\right]^{\rho_{4}(N, \beta)},\left[\rho_{5}(\varepsilon, N)\right]^{\rho_{6}(N, \beta)}\right\} .
$$

Then there exists a positive constant $\tilde{C}=\tilde{C}\left(u_{0} ; \gamma_{1}, \gamma_{2}, C_{0}, T, \Omega\right)$ such that the solution of (25)-(26) satisfies the following error estimate for $\Pi^{m}:=$ $u\left(t_{m}\right)-u^{m}$,

$$
\begin{aligned}
& \max _{0 \leq m \leq M}\left\|\Pi^{m}\right\|_{H^{-1}}+\left(k \sum_{m=1}^{M}\left\{k\left\|d_{t} \Pi^{m}\right\|_{H^{-1}}^{2}+k^{\beta}\left\|\nabla \Pi^{m}\right\|_{L^{2}}^{2}\right\}\right)^{\frac{1}{2}} \\
& \quad \leq \tilde{C} k^{\frac{1-\beta}{2}}\left[\rho_{2}(\varepsilon)\right]^{\frac{1}{2}} .
\end{aligned}
$$

Proof. The proof is split into four steps: the first step deals with consistency error and shows the relevancy of the condition $\left(\mathrm{GA}_{1}\right)_{3}$ imposed on $f$. Steps two and three use Proposition 1 and stability properties of the implicit Euler-method to avoid exponential blow-up in $\varepsilon^{-1}$ of the error constant. In the last step, an inductive argument is used to handle the difficulty caused by the super-quadratic term in $\left(\mathrm{GA}_{1}\right)_{3}$. 
Step 1. Let $e^{m}:=u\left(t_{m}\right)-u^{m} \in L_{0}^{2}(\Omega)$ and $g^{m}:=w\left(t_{m}\right)-w^{m}$ denote the error functions. From (25)-(26) we get error equations

$$
\begin{aligned}
\left(d_{t} e^{m+1}, \eta\right)+\left(\nabla g^{m+1}, \nabla \eta\right) & =\left(\mathcal{R}\left(u_{t t} ; m\right), \eta\right), \\
\text { (39) } \varepsilon\left(\nabla e^{m+1}, \nabla v\right)+\frac{1}{\varepsilon}\left(f\left(u\left(t_{m}\right)\right)-f\left(u^{m+1}\right), v\right) & =\left(g^{m+1}, v\right),
\end{aligned}
$$

which are valid for all $(\eta, v) \in\left[H^{1}(\Omega)\right]^{2}$, and

$$
\mathcal{R}\left(u_{t t} ; m\right)=-\frac{1}{k} \int_{t_{m-1}}^{t_{m}}\left(s-t_{m-1}\right) u_{t t}(s) \mathrm{d} s .
$$

We choose $(\eta, v)=\left(-\Delta^{-1} e^{m+1}, e^{m+1}\right)$ and find

$$
\text { (41) } \begin{aligned}
\frac{1}{2} & d_{t}\left\|\Delta^{-\frac{1}{2}} e^{m+1}\right\|_{L^{2}}^{2}+\frac{k}{2}\left\|\Delta^{-\frac{1}{2}} d_{t} e^{m+1}\right\|_{L^{2}}^{2}+\varepsilon\left\|\nabla e^{m+1}\right\|_{L^{2}}^{2} \\
& +\frac{1}{\varepsilon}\left(f\left(u\left(t_{m+1}\right)\right)-f\left(u^{m+1}\right), e^{m+1}\right)=\left(-\Delta^{-1} \mathcal{R}\left(u_{t t} ; m\right), e^{m+1}\right) .
\end{aligned}
$$

From (ix) of Lemma 1,

$$
\begin{aligned}
& k \sum_{m=0}^{M}\left\|\Delta^{-1} \mathcal{R}\left(u_{t t} ; m\right)\right\|_{H^{-1}}^{2} \\
& \quad \leq \frac{1}{k} \sum_{m=0}^{M}\left[\int_{t_{m}}^{t_{m+1}} \frac{\left(s-t_{m}\right)^{2}}{\tau(s)} \mathrm{d} s\right]\left[\int_{t_{m}}^{t_{m+1}} \tau(s)\left\|\Delta^{-1} u_{t t}(s)\right\|_{H^{-1}}^{2} \mathrm{~d} s\right] \\
& \quad \leq C k \rho_{2}(\varepsilon) .
\end{aligned}
$$

To control the last term on the left hand side of (41), we use $\left(\mathrm{GA}_{1}\right)_{3}$,

$$
\frac{1}{\varepsilon}\left(f\left(u\left(t_{m}\right)\right)-f\left(u^{m}\right), e^{m}\right) \geq \frac{\gamma_{1}}{\varepsilon}\left(f^{\prime}\left(u\left(t_{m}\right)\right) e^{m}, e^{m}\right)-\frac{\gamma_{2}}{\varepsilon}\left\|e^{m}\right\|_{L^{2+\delta}}^{2+\delta} .
$$

Step 2. We want to use the following spectrum estimate result (see Proposition 1) to bound from below the first term on the right hand side of (43)

(44) $\varepsilon\|\nabla \phi\|_{L^{2}}^{2}+\frac{1}{\varepsilon}\left(f^{\prime}(u) \phi, \phi\right) \geq-C_{0}\left\|\Delta^{-\frac{1}{2}} \phi\right\|_{L^{2}}^{2}, \quad \forall \phi \in H^{1}(\Omega)$,

where $C_{0}>0$ is independent of $\varepsilon$. At the same time, we want to make use of the $H^{-1}(\Omega)$ norm of $-\Delta^{-1} \mathcal{R}\left(u_{t t} ; m\right)$ in order to keep the power of $\frac{1}{\varepsilon}$ as low as possible in the error constant. The latter requires to keep portions of $\left\|\nabla e^{m+1}\right\|_{L^{2}}^{2}$ on the left hand side of the error equation (41). To this end, we 
apply (44) with a scaling factor $\gamma_{1}\left(1-\frac{k^{\beta}}{2}\right)$, which together with (43) and (41) gives

$$
\begin{aligned}
& \frac{1}{2} d_{t}\left\|\Delta^{-\frac{1}{2}} e^{m+1}\right\|_{L^{2}}^{2}+\frac{k}{2}\left\|\Delta^{-\frac{1}{2}} d_{t} e^{m+1}\right\|_{L^{2}}^{2} \\
& +\frac{\varepsilon}{2}\left[1-\gamma_{1}\left(1-\frac{k^{\beta}}{2}\right)\right]\left\|\nabla e^{m+1}\right\|_{L^{2}}^{2} \\
& \leq C_{0} \gamma_{1}\left(1-\frac{k^{\beta}}{2}\right)\left\|\Delta^{-\frac{1}{2}} e^{m+1}\right\|_{L^{2}}^{2}-\frac{\gamma_{1} k^{\beta}}{2 \varepsilon}\left(f^{\prime}\left(u\left(t_{m+1}\right)\right) e^{m+1}, e^{m+1}\right) \\
& +\frac{C}{\varepsilon}\left\|e^{m+1}\right\|_{L^{2+\delta}}^{2+\delta}+C \frac{k^{-\beta}}{\varepsilon}\left\|\Delta^{-1} \mathcal{R}\left(u_{t t} ; m\right)\right\|_{H^{-1}}^{2} .
\end{aligned}
$$

From (15), the second term on the right hand side can be bounded as

$$
\begin{gathered}
-\frac{\gamma_{1} k^{\beta}}{2 \varepsilon}\left(f^{\prime}\left(u\left(t_{m+1}\right)\right) e^{m+1}, e^{m+1}\right) \leq \tilde{c}_{0} \frac{\gamma_{1} k^{\beta}}{2 \varepsilon}\left\|e^{m+1}\right\|_{L^{2}}^{2} \\
\quad \leq \tilde{c}_{0} \frac{\gamma_{1} k^{\beta}}{8 \varepsilon^{3}}\left\|\Delta^{-\frac{1}{2}} e^{m+1}\right\|_{L^{2}}^{2}+\frac{\gamma_{1} \varepsilon k^{\beta}}{4}\left\|\nabla e^{m+1}\right\|_{L^{2}}^{2} .
\end{gathered}
$$

Then, we obtain from (42) and (46) after summing (45) over $m$ from 0 to $\ell(\leq M)$

$$
\begin{aligned}
& \frac{1}{2}\left\|\Delta^{-\frac{1}{2}} e^{\ell+1}\right\|_{L^{2}}^{2}+k \sum_{m=0}^{\ell}\left\{\frac{k}{2}\left\|\Delta^{-\frac{1}{2}} d_{t} e^{m+1}\right\|_{L^{2}}^{2}\right. \\
& \left.\quad+\frac{\varepsilon}{4}\left[1-\gamma_{1}\left(1-\frac{k^{\beta}}{2}\right)\right]\left\|\nabla e^{m+1}\right\|_{L^{2}}^{2}\right\} \\
& \leq\left(C_{0} \gamma_{1}+\frac{\tilde{c}_{0} \gamma_{1} k^{\beta}}{\varepsilon^{3}}\right) k \sum_{m=1}^{\ell+1}\left\|\Delta^{-\frac{1}{2}} e^{m}\right\|_{L^{2}}^{2}+C k^{1-\beta} \rho_{2}(\varepsilon) \\
& \quad+\frac{C k}{\varepsilon} \sum_{m=1}^{\ell+1}\left\|e^{m}\right\|_{L^{2+\delta}}^{2+\delta} .
\end{aligned}
$$

Note that $k=O\left(\varepsilon^{\frac{3}{\beta}}\right)$ in the coefficient of the first term on the right hand side in order to avoid exponential growth in $\frac{1}{\varepsilon}$ of the stability constraint arising from discrete Gronwall's inequality.

Step 3. We now need to bound the super-quadratic term at the end of the inequality (47). First, a shift in the super-index leads to

$$
\frac{1}{\varepsilon}\left\|e^{m+1}\right\|_{L^{2+\delta}}^{2+\delta} \leq \frac{C}{\varepsilon}\left(\left\|e^{m}\right\|_{L^{2+\delta}}^{2+\delta}+k^{2+\delta}\left\|d_{t} e^{m+1}\right\|_{L^{2+\delta}}^{2+\delta}\right) .
$$


For the first term on the right hand side, we interpolate $L^{2+\delta}$ between $L^{2}$ and $H^{2}$, and using (v) of Lemma 1 and (v) of Lemma 3, we infer

$$
\begin{aligned}
& \frac{1}{\varepsilon}\left\|e^{m}\right\|_{L^{2+\delta}}^{2+\delta} \leq \frac{C}{\varepsilon}\left(\left\|\Delta e^{m}\right\|_{L^{2}}^{\frac{N \delta}{4}}\left\|e^{m}\right\|_{L^{2}}^{\frac{8+(4-N) \delta}{4}}+\left\|e^{m}\right\|_{L^{2}}^{2+\delta}\right) \\
& \leq \frac{C}{\varepsilon}\left\|e^{m}\right\|_{L^{2}}^{\frac{8+(4-N) \delta}{4}}\left(\left\|\Delta e^{m}\right\|_{L^{2}}^{\frac{N \delta}{4}}+\left\|e^{m}\right\|_{L^{2}}^{\frac{N \delta}{4}}\right) \\
& \quad \leq \frac{C}{\varepsilon} \rho_{1}(\varepsilon)^{\frac{N \delta}{8}}\left\|e^{m}\right\|_{L^{2}}^{\frac{8+(4-N) \delta}{8}} .
\end{aligned}
$$

Since $\int_{\Omega} e^{m} \mathrm{~d} x=0$, the above inequality is continued by

$$
\frac{1}{\varepsilon}\left\|e^{m}\right\|_{L^{2+\delta}}^{2+\delta} \leq C\left[\frac{1}{\gamma} \rho_{1}(\varepsilon)^{\frac{N \delta}{8}}\left\|\Delta^{-\frac{1}{2}} e^{m}\right\|_{L^{2}}^{\frac{8+(4-N) \delta}{8}}\right]\left[\gamma\left\|\nabla e^{m}\right\|_{L^{2}}^{\frac{8+(4-N) \delta}{8}}\right]
$$

for some $\gamma>0$ to be fixed in the sequel.

The subsequent analysis deals with $0<\delta<\frac{8}{4-N}$, which is the more involved case. It is crucial to recover a super-quadratic exponent for $\left\|\Delta^{-\frac{1}{2}} e^{m}\right\|_{L^{2}}$ in Step 4.

We come back to (50) and look for $\alpha>0$ such that

$$
\gamma^{\alpha}\left\|\nabla e^{m}\right\|_{L^{2}}^{\frac{8+(4-N) \delta}{8} \alpha} \leq \frac{\varepsilon}{4}\left[1-\gamma_{1}\left(1-\frac{k^{\beta}}{2}\right)\right]\left\|\nabla e^{m}\right\|_{L^{2}}^{2},
$$

which implies $\frac{8+(4-N) \delta}{8} \alpha=2$, or $\alpha=\frac{16}{8+(4-N) \delta}$; moreover we set $\gamma^{-1}=$ $C \varepsilon^{-\frac{1}{\alpha}} k^{-\frac{\beta}{\alpha}}$. We use these choices in (50), together with Young's inequality, to find after a short calculation that (50) is continued by

$$
\begin{gathered}
\text { (51) } \frac{1}{\varepsilon}\left\|e^{m}\right\|_{L^{2+\delta}}^{2+\delta} \leq C \\
{\left[\varepsilon k^{\beta}\right]^{-\frac{8+(4-N) \delta}{8-(4-N) \delta}} \rho_{1}(\varepsilon)^{\frac{2 N \delta}{8-(4-N) \delta}}\left\|\Delta^{-\frac{1}{2}} e^{m}\right\|_{L^{2}}^{2\left(1+\frac{(4-N) \delta}{8-(4-N) \delta}\right)}} \\
+\frac{\varepsilon}{8}\left[1-\gamma_{1}\left(1-\frac{k^{\beta}}{2}\right)\right]\left\|\nabla e^{m}\right\|_{L^{2}}^{2} .
\end{gathered}
$$

Similarly, the second term on the right hand side of (48) can be bounded as

$$
\begin{aligned}
& \frac{k^{2+\delta}}{\varepsilon}\left\|d_{t} e^{m+1}\right\|_{L^{2+\delta}}^{2+\delta} \\
\leq & \frac{C k^{2+\delta}}{\varepsilon}\left(\left\|\Delta d_{t} e^{m+1}\right\|_{L^{2}}^{\frac{N \delta}{4}}\left\|d_{t} e^{m+1}\right\|_{L^{2}}^{\frac{8+(4-N) \delta}{4}}+\left\|d_{t} e^{m+1}\right\|_{L^{2}}^{2+\delta}\right) \\
\leq & \frac{C k^{2+\delta}}{\varepsilon}\left\|d_{t} e^{m+1}\right\|_{L^{2}}^{\frac{8+(4-N) \delta}{4}}\left(\left\|\Delta d_{t} e^{m+1}\right\|_{L^{2}}^{\frac{N \delta}{4}}+\left\|d_{t} e^{m+1}\right\|_{L^{2}}^{\frac{N \delta}{4}}\right) \\
\leq & C k^{2+\frac{(4-N) \delta}{4}} \rho_{1}(\varepsilon)^{\frac{N \delta}{8}}\left\|d_{t} e^{m+1}\right\|_{L^{2}}^{2 \frac{8+(4-N) \delta}{8}} \\
\leq & C\left[\frac{1}{\gamma} k^{2+\frac{(4-N) \delta}{4}} \rho_{1}(\varepsilon)^{\frac{N \delta}{8}}\left\|\nabla d_{t} e^{m+1}\right\|_{L^{2}}^{\frac{8+(4-N) \delta}{8}}\right]\left[\gamma\left\|\Delta^{-\frac{1}{2}} d_{t} e^{m+1}\right\|_{L^{2}}^{\frac{8+(4-N) \delta}{8}}\right] .
\end{aligned}
$$


We look for $\alpha>0$ such that

$$
\gamma^{\alpha}\left\|\Delta^{-\frac{1}{2}} d_{t} e^{m+1}\right\|_{L^{2}}^{\frac{8+(4-N) \delta}{8} \alpha} \leq \frac{k}{4}\left\|\Delta^{-\frac{1}{2}} d_{t} e^{m+1}\right\|_{L^{2}}^{2} .
$$

Then $\frac{8+(4-N) \delta}{8} \alpha=2$ implies $\alpha=\frac{16}{8+(4-N) \delta}$, and also $\gamma=k^{\frac{8+(4-N) \delta}{16}}$. Hence, an upper bound for (52) is

$$
\begin{aligned}
\frac{k}{4} \| & \Delta^{-\frac{1}{2}} d_{t} e^{m+1} \|_{L^{2}}^{2}+C k^{\left(\frac{8+(4-N) \delta}{4}-\frac{8-(4-N) \delta}{16}\right) \frac{16}{8-(4-N) \delta}} \\
& \times \rho_{1}(\varepsilon)^{\frac{2 N \delta}{8-(4-N) \delta}}\left\|\nabla d_{t} e^{m+1}\right\|_{L^{2}}^{2 \frac{8+(4-N) \delta}{8-N) \delta}} \\
= & C k^{3 \frac{8+(4-N) \delta}{8-(4-N) \delta}} \rho_{1}(\varepsilon)^{\frac{2 N \delta}{8-(4-N) \delta}}\left\|\nabla d_{t} e^{m+1}\right\|_{L^{2}}^{2 \frac{8+(4-N) \delta}{8-(4-N) \delta}}+\frac{k}{4}\left\|\Delta^{-\frac{1}{2}} d_{t} e^{m+1}\right\|_{L^{2}}^{2} \\
\leq & C k^{3 \frac{8+(4-N) \delta}{8-(4-N) \delta}-2 \frac{(4-N) \delta}{8-(4-N) \delta}} \rho_{1}(\varepsilon)^{\frac{2 N \delta}{8-(4-N) \delta}} \\
& \times \max _{i=0,1}\left\{\left\|\nabla e^{m+i}\right\|_{L^{2} \frac{(4-N) \delta}{8-(4-N) \delta}}\right\}\left\|\nabla d_{t} e^{m+1}\right\|_{L^{2}}^{2}+\frac{k}{4}\left\|\Delta^{-\frac{1}{2}} d_{t} e^{m+1}\right\|_{L^{2}}^{2} \\
\leq & C k^{3+4 \frac{(4-N) \delta}{8-(4-N) \delta}} \varepsilon^{-\frac{\left(2 \sigma_{1}+1\right)(4-N) \delta}{8-(4-N) \delta}} \rho_{1}(\varepsilon)^{\frac{2 N \delta}{8-(4-N) \delta}}\left\|\nabla d_{t} e^{m+1}\right\|_{L^{2}}^{2} \\
& +\frac{k}{4}\left\|\Delta^{-\frac{1}{2}} d_{t} e^{m+1}\right\|_{L^{2}}^{2} .
\end{aligned}
$$

Finally, combining these results with (47), and using (vi) of Lemma 1 and (ii) of 3 we get

$$
\begin{aligned}
\frac{1}{2}\left\|\Delta^{-\frac{1}{2}} e^{\ell+1}\right\|_{L^{2}}^{2}+k \sum_{m=0}^{\ell}\left\{\frac{k}{2}\left\|\Delta^{-\frac{1}{2}} d_{t} e^{m+1}\right\|_{L^{2}}^{2}\right. \\
\left.\quad+\frac{\varepsilon}{8}\left[1-\gamma_{1}\left(1-\frac{k^{\beta}}{2}\right)\right]\left\|\nabla e^{m+1}\right\|_{L^{2}}^{2}\right\} \\
\leq\left(C_{0} \gamma_{1}+\tilde{c}_{0} \gamma_{1} k^{\beta} \varepsilon^{-3}\right) k \sum_{m=0}^{\ell}\left\|\Delta^{-\frac{1}{2}} e^{m+1}\right\|_{L^{2}}^{2}+C k^{1-\beta} \rho_{2}(\varepsilon) \\
+C\left[\varepsilon k^{\beta}\right]^{-\frac{8+(4-N) \delta}{8-(4-N) \delta}} \rho_{1}(\varepsilon)^{\frac{2 N \delta}{8-(4-N) \delta}} k \sum_{m=0}^{\ell}\left\|\Delta^{-\frac{1}{2}} e^{m}\right\|_{L^{2}}^{2\left(1+\frac{(4-N) \delta}{8-(4-N) \delta}\right)} \\
+C k^{2+4 \frac{(4-N) \delta}{8-(4-N) \delta}} \varepsilon^{-\left\{\frac{\left(2 \sigma_{1}+1\right)(4-N) \delta}{8-(4-N) \delta}+2\left(\sigma_{1}+2\right)\right\}} \rho_{1}(\varepsilon)^{\frac{2 N \delta}{8-(4-N) \delta}} .
\end{aligned}
$$

Step 4. We now conclude the proof by the following induction argument which is based on the results from Steps 1 to 3. Suppose that for sufficiently small time steps satisfying

$$
k \leq \tilde{C} \min \left\{\varepsilon^{\frac{3}{\beta}}, \varepsilon^{\alpha_{0}},\left[\rho_{3}(\varepsilon)\right]^{\rho_{4}(N, \beta)},\left[\rho_{5}(\varepsilon, N)\right]^{\rho_{6}(N, \beta)}\right\}
$$


and $0<\beta<\frac{1}{2}$, there exist two positive constants

$$
c_{1}=c_{1}\left(t_{\ell}, \Omega, u_{0}, \sigma_{i}, p\right), \quad c_{2}=c_{2}\left(t_{\ell}, \Omega, u_{0}, \sigma_{i}, p ; C_{0}\right),
$$

independent of $k$ and $\varepsilon$, such that the following inequality holds

$$
\begin{aligned}
& \max _{0 \leq m \leq \ell} \frac{1}{2}\left\|\Delta^{-\frac{1}{2}} e^{m}\right\|_{L^{2}}^{2}+k \sum_{m=1}^{\ell}\left(\frac{k}{2}\left\|\Delta^{-\frac{1}{2}} d_{t} e^{m}\right\|_{L^{2}}^{2}+\frac{\gamma_{1} \varepsilon k^{\beta}}{2}\left\|\nabla e^{m}\right\|_{L^{2}}^{2}\right) \\
& \leq c_{1} k^{1-\beta} \rho_{2}(\varepsilon) \exp \left(c_{2} t_{\ell}\right) .
\end{aligned}
$$

The last two constraints in (54) arise as follows. The first of them comes from controlling the last error term in (53)

$$
\begin{aligned}
& k^{2+4 \frac{(4-N) \delta}{8-(4-N) \delta}} \varepsilon^{-\left\{\frac{\left(2 \sigma_{1}+1\right)(4-N) \delta}{8-(4-N) \delta}+2\left(\sigma_{1}+2\right)\right\}}\left[\rho_{1}(\varepsilon)\right]^{\frac{2 N \delta}{8-(4-N) \delta}} \\
& \quad \leq \frac{c_{1}}{2} k^{1-\beta} \rho_{2}(\varepsilon) \exp \left(c_{2} t_{\ell}\right) .
\end{aligned}
$$

Note that the exponent in the second sum on the right hand side of (53) is bigger than 2 , hence we can recover $(55)$ at the $(\ell+1)$ th time step by using the discrete Gronwall's inequality, provided that

$$
\begin{aligned}
& {\left[\varepsilon k^{\beta}\right]^{-\frac{8+(4-N) \delta}{8-(4-N) \delta}} \rho_{1}(\varepsilon)^{\frac{2 N \delta}{8-(4-N) \delta}}\left[k^{1-\beta} \rho_{2}(\varepsilon)\right]^{1+\frac{(4-N) \delta}{8-(4-N) \delta}}} \\
& \quad \leq \frac{c_{1}}{2} k^{1-\beta} \rho_{2}(\varepsilon) \exp \left(c_{2} t_{\ell+1}\right)
\end{aligned}
$$

which gives the last constraint in (54). The proof is complete.

Remark (a). Theorem 1 is stated for $0<\delta<\frac{8}{4-N}$, which covers assumption $\left(\mathrm{GA}_{1}\right)$ for the case $N=2,3$. For $N=2$, the error estimate is valid for all $0<\delta<\infty$, the above proof can be adapted and simplified for the case $\delta>\frac{8}{4-N}$. Note that in this case the crucial requirement of super-quadratical growth is already met in (50), then we can immediately jump to Step 4 to proceed. Finally, the case $\delta=\frac{8}{4-N}$ is again easy to take care, thanks to Lemma 1 and 3.

(b). In addition to the spectrum estimate of Proposition 1, the stability estimate (v) of Lemma 3 is critical to the analysis.

(c). It is natural to estimate the error of (25)-(26) in the norm of $\ell^{\infty}$ $\left(J_{k}^{1} ; H^{-1}(\Omega)\right) \cap \ell^{2}\left(J_{k}^{1} ; H^{1}(\Omega)\right)$, its structure allows to test with $-\Delta^{-1}$ $e^{m+1}$, which then interferes with limited regularity property of $u_{t t}$ and cuts convergence rate in (42) down to sub-optimal order. As to be demonstrated in the sequel, using stretched meshes $J_{k}^{2}$ will help to attain a quasi-optimal order for the Euler method (25)-(26). 
(d). A straightforward idea to benefit from the damping property of (1) is to multiply (41) by a time-weight $\tau_{m+1}:=\min \left\{t_{m+1}, 1\right\}$ before summation; this would give an optimal convergence rate $C k^{2} \rho_{2}(\varepsilon)$ in (42) thanks to (ix) of Lemma 1. On the other hand, this would require to control the error $\left\{e^{m}\right\}_{m=0}^{M}$ in the norm of $\ell^{2}\left(J_{k}^{1} ; H^{-1}(\Omega)\right)$ by using a (parabolic) duality argument. This strategy does not seem to be successful in the present analysis where we focus on non-exponential dependencies on $\frac{1}{\epsilon}$ of involved stability constants.

(e). It is clear that the smaller $\beta$, the better the error bound, since the exponent of $k$ is closer to $\frac{1}{2}$. Small values of $\beta$, however, restrict admissible time steps to small sizes.

(f). The proof of Theorem 1 suggests the following numerical stabilization technique for the Cahn-Hilliard equation (25)-(26)

$$
\begin{aligned}
& \left(d_{t} u^{m+1}, \eta\right)+\left(\nabla w^{m+1}, \nabla \eta\right)=0 \quad \forall \eta \in H^{1}(\Omega), \\
& \varepsilon\left(1+\frac{k^{\zeta 1}}{\varepsilon^{\zeta 2}}\right)\left(\nabla u^{m+1}, \nabla v\right)+\frac{1}{\varepsilon}\left(f\left(u^{m+1}\right), v\right) \\
& \quad=\left(w^{m+1}, v\right) \quad \forall v \in H^{1}(\Omega),
\end{aligned}
$$

where $\zeta_{i} \geq 0$ for $i=1,2$. We will not go into further discussion of these methods here.

For given more regular initial datum $u_{0} \in H^{3}(\Omega)$ and domain $\Omega$ (see assumptions in Lemma 2), the convergence rate can be improved to $C k^{1-\beta}$ $\rho_{2}(\varepsilon)$. The key ingredient for proving that is to use (iv) of Lemma 2 to improve on the estimate (42).

Corollary 1 Let $\left\{\left(u^{m}, w^{m}\right\}_{m=0}^{M}\right.$ solve (25)-(26) on an equidistant mesh $J_{k}^{1}=$ $\left\{t_{m}\right\}_{m=0}^{M}$ of mesh size $O(k)$, for $u_{0} \in H^{3}(\Omega)$, and $\partial \Omega$ of class $C^{2,1}$ (or a convex polygonal domain when $N=2)$. Suppose $\left(G A_{1}\right)-\left(G A_{3}\right)$ hold, and $1<\delta<\frac{8}{4-N}$. Let $\rho_{j}$ be same as in Theorem 1. For fixed positive values $0<\beta<\frac{1}{2}$, let $k$ satisfy the following constraint

$$
k \leq \tilde{C} \min \left\{\varepsilon^{\frac{3}{\beta}}, \varepsilon^{\alpha_{0}},\left[\rho_{3}(\varepsilon, N)\right]^{\rho_{4}(N, \beta)},\left[\rho_{5}(\varepsilon, N)\right]^{\rho_{6}(N, \beta)}\right\},
$$

Then there exists a positive constant $\tilde{C}=\tilde{C}\left(u_{0} ; \gamma_{1}, \gamma_{2}, C_{0}, T, \Omega\right)$ such that the solution of (25)-(26) satisfies the following error estimate, for $\Pi^{m}:=$ $u\left(t_{m}\right)-u^{m}$,

$$
\begin{aligned}
& \max _{0 \leq m \leq M}\left\|\Pi^{m}\right\|_{H^{-1}}+\left(k \sum_{m=1}^{M}\left\{k\left\|d_{t} \Pi^{m}\right\|_{H^{-1}}^{2}+k^{\beta}\left\|\nabla \Pi^{m}\right\|_{L^{2}}^{2}\right\}\right)^{\frac{1}{2}} \\
& \quad \leq \tilde{C} k^{\frac{2-\beta}{2}}\left[\rho_{2}(\varepsilon)\right]^{\frac{1}{2}} .
\end{aligned}
$$


For $u_{0} \in H^{2}(\Omega)$, the error bound given in Theorem 1 is not optimal, the crucial step where we loose accuracy by one order of magnitude on the time step $k$ is (42), since we are only provided with a bound for $\sqrt{\tau}(\Delta)^{-1} u_{t t} \in$ $L^{2}\left(J ; H^{-1}(\Omega)\right)$; see (ix) of Lemma 1 . The following result reflects the stabilizing effect of the mesh $J_{k}^{2}$ in this respect. Note that the proof of Theorem 1 only requires (iv)-(v) of Lemma 3 which are replaced by (vii) in the case of the mesh $J_{k}^{2}$.

Theorem 2 Suppose that the assumptions and shorthand notation of Theorem 1 hold. Define

$$
\begin{aligned}
& \tilde{\rho}_{4}(N, \beta)=\left[\beta+\frac{4(4-N) \delta}{8-(4-N) \delta}\right]^{-1}, \\
& \tilde{\rho}_{6}(N, \beta)=\delta\left[2(4-N) \delta-\beta(16+3(4-N) \delta]^{-1} .\right.
\end{aligned}
$$

For fixed positive values $0<\beta<\frac{1}{2}$, let $k_{0}$ satisfy the following constraint

$$
k_{0} \leq \tilde{C} \min \left\{\varepsilon^{\frac{3}{\beta}}, \varepsilon^{\frac{\alpha_{0}}{2}},\left[\rho_{3}(\varepsilon, N)\right]^{\tilde{\rho}_{4}(N, \beta)},\left[\rho_{5}(\varepsilon, N)\right]^{\tilde{\rho}_{6}(N, \beta)}\right\} .
$$

Let $\left\{\left(u^{m}, w^{m}\right)\right\}_{m=0}^{M}$ be the solution to (25)-(26) on the mesh $J_{k}^{2}$. Then there holds the following improved error estimate for $\Pi^{m}:=u\left(t_{m}\right)-u^{m}$,

$$
\begin{aligned}
& \max _{0 \leq m \leq M}\left\|\Pi^{m}\right\|_{H^{-1}}+\left(\sum_{m=1}^{M} k_{m}\left\{k_{m}\left\|d_{t} \Pi^{m}\right\|_{H^{-1}}^{2}+k_{m}^{\beta}\left\|\nabla \Pi^{m}\right\|_{L^{2}}^{2}\right\}\right)^{\frac{1}{2}} \\
& \quad \leq \tilde{C} k_{0}^{\frac{2-\beta}{2}}\left[\rho_{2}(\varepsilon)\right]^{\frac{1}{2}} .
\end{aligned}
$$

Proof. The proof follows the steps of that of Theorem 1. We only sketch the necessary modifications in the following.

Step 1 On the stretched mesh $J_{k}^{2}$, the residual $\mathcal{R}\left(u_{t t}, m\right)$ can be bounded as follows

$$
\begin{aligned}
& \sum_{m=1}^{M} k_{m+1}\left\|\Delta^{-1} \mathcal{R}\left(u_{t t}, m\right)\right\|_{H^{-1}}^{2}=\sum_{m=1}^{M} \frac{1}{k_{m+1}} \\
& \quad\left\|\int_{t_{m}}^{t_{m+1}} \times\left(s-t_{m}\right) \Delta^{-1} u_{t t}(s) \mathrm{d} s\right\|_{H^{-1}}^{2} \\
& \quad \leq \sum_{m=1}^{M} \frac{1}{k_{m+1}} \int_{t_{m}}^{t_{m+1}} \frac{1}{s}\left(s-t_{m}\right)^{2} \mathrm{~d} s \int_{t_{m}}^{t_{m+1}} \tau(s)\left\|\Delta^{-1} u_{t t}(s)\right\|_{H^{-1}}^{2} \mathrm{~d} s \\
& \quad \leq C \max _{0 \leq m \leq M}\left\{\frac{1}{k_{m+1}} \int_{t_{m}}^{t_{m+1}} \frac{1}{s}\left(s-t_{m}\right)^{2} \mathrm{~d} s\right\} \rho_{2}(\varepsilon) \\
& \quad \leq C \max _{1 \leq m \leq M} \frac{k_{m}^{2}}{t_{m}} \rho_{2}(\varepsilon) \leq C k_{0}^{4} \max _{1 \leq m \leq M} \frac{(m+1)^{2}}{t_{m}} \rho_{2}(\varepsilon) \leq C k_{0}^{2} \rho_{2}(\varepsilon),
\end{aligned}
$$


thanks to (ix) of Lemma 1. This improved upper bound for the residual replaces (42) in the proof of Theorem 1.

Step 2 This step is the same.

Step 3 We use (vii) of Lemma 3, instead of (v) to bound $\max _{0 \leq m \leq M}\left\|\Delta u^{m}\right\|_{L^{2}}$. Then (50) and (51) are replaced by

$$
\text { (60) } \begin{aligned}
\frac{1}{\varepsilon}\left\|e^{m}\right\|_{L^{2+\delta}}^{2+\delta} \leq & C\left[\varepsilon k_{m}^{\beta}\right]^{-\frac{8+(4-N) \delta}{8-(4-N) \delta}} \tilde{\rho}_{1}(\varepsilon)^{\frac{2 N \delta}{8-(4-N) \delta}}\left\|\Delta^{-\frac{1}{2}} e^{m}\right\|_{L^{2}}^{2\left(1+\frac{(4-N) \delta}{8-(4-N) \delta}\right)} \\
& +\frac{\varepsilon}{8}\left[1-\gamma_{1}\left(1-\frac{k_{m}^{\beta}}{2}\right)\right]\left\|\nabla e^{m}\right\|_{L^{2}}^{2} .
\end{aligned}
$$

Again, the argument applies for values $\delta<\frac{8}{4-N}$. Instead of (52), we now have

$$
\begin{aligned}
& \frac{k_{m+1}^{2+\delta}}{\varepsilon}\left\|d_{t} e^{m+1}\right\|_{L^{2+\delta}}^{2+\delta} \\
& \leq \frac{C k_{m+1}^{2+\delta}}{\varepsilon}\left(\left\|\Delta d_{t} e^{m+1}\right\|_{L^{2}}^{\frac{N \delta}{4}}\left\|d_{t} e^{m+1}\right\|_{L^{2}}^{\frac{8+(4-N) \delta}{4}}+\left\|d_{t} e^{m+1}\right\|_{L^{2}}^{2+\delta}\right) \\
& \leq \frac{C k_{m+1}^{2+\delta}}{\varepsilon}\left\|d_{t} e^{m+1}\right\|_{L^{2}}^{\frac{8+(4-N) \delta}{4}}\left(\left\|\Delta d_{t} e^{m+1}\right\|_{L^{2}}^{\frac{N \delta}{4}}+\left\|d_{t} e^{m+1}\right\|_{L^{2}}^{\frac{N \delta}{4}}\right) \\
& \leq C k_{m+1}^{3+4 \frac{(4-N) \delta}{8-(4-N) \delta}} \varepsilon^{-\frac{\left(2 \sigma_{1}+1\right)(4-N) \delta}{8-(4-N) \delta}} \rho_{1}(\varepsilon)^{\frac{2 N \delta}{8-(4-N) \delta}}\left\|\nabla d_{t} e^{m+1}\right\|_{L^{2}}^{2} \\
& \quad+\frac{k_{m+1}}{4}\left\|\Delta^{-\frac{1}{2}} d_{t} e^{m+1}\right\|_{L^{2}}^{2} .
\end{aligned}
$$

Finally, (53) is replaced by

$$
\begin{aligned}
& \frac{1}{2}\left\|\Delta^{-\frac{1}{2}} e^{\ell}\right\|_{L^{2}}^{2}+\sum_{m=1}^{\ell}\left\{\frac{k_{m}^{2}}{2}\left\|\Delta^{-\frac{1}{2}} d_{t} e^{m}\right\|_{L^{2}}^{2}+\frac{\varepsilon k_{m}}{8}\left[1-\gamma_{1}\left(1-\frac{k_{m}^{\beta}}{2}\right)\right]\left\|\nabla e^{m}\right\|_{L^{2}}^{2}\right\} \\
& \leq\left(C_{0} \gamma_{1}+\tilde{c}_{0} \gamma_{1} k_{0}^{\beta} \varepsilon^{-3}\right) \sum_{m=1}^{\ell} k_{m}\left\|\Delta^{-\frac{1}{2}} e^{m}\right\|_{L^{2}}^{2}+C k_{0}^{2-\beta} \rho_{2}(\varepsilon) \\
& d+C\left[\tilde{\rho}_{1}(\varepsilon)\right]^{\frac{2 N \delta}{8-(4-N) \delta}} \sum_{m=1}^{\ell} k_{m}\left[\varepsilon k_{m}^{\beta}\right]^{-\frac{8+(4-N) \delta}{8-(4-N) \delta}}\left\|\Delta^{-\frac{1}{2}} e^{m}\right\|_{L^{2}}^{2\left(1+\frac{(4-N) \delta}{8-(4-N) \delta}\right)} \\
& \quad+C k_{0}^{2+4 \frac{(4-N) \delta}{8-(4-N) \delta}} \varepsilon^{-\left\{\frac{\left(2 \sigma_{1}+1\right)(4-N) \delta}{8-(4-N) \delta}+2\left(\sigma_{1}+2\right)\right\}}\left[\tilde{\rho}_{1}(\varepsilon)\right]^{\frac{2 N \delta}{8-(4-N) \delta}} .
\end{aligned}
$$

Step 4 The inductive argument now reads: Suppose that for sufficiently small basic time step $k_{0}$ satisfying

$$
k_{0} \leq \tilde{C} \min \left\{\varepsilon^{\frac{3}{\beta}}, \varepsilon^{\alpha_{0}},\left[\rho_{3}(\varepsilon)\right]^{\tilde{\rho}_{4}(N, \beta)},\left[\rho_{5}(\varepsilon, N)\right]^{\tilde{\rho}_{6}(N, \beta)}\right\}
$$


and $0<\beta<\frac{1}{2}$, there exist two positive constants

$$
\tilde{c}_{1}=\tilde{c}_{1}\left(t_{\ell}, \Omega, u_{0}, \sigma_{i}, p\right), \quad \tilde{c}_{2}=\tilde{c}_{2}\left(t_{\ell}, \Omega, u_{0}, \sigma_{i}, p ; C_{0}\right),
$$

independent of $k_{0}$ and $\varepsilon$, such that the following inequality holds,

$$
\text { (63) } \begin{aligned}
& \max _{0 \leq m \leq \ell}\left\|\Delta^{-\frac{1}{2}} e^{m}\right\|_{L^{2}}^{2}+\sum_{m=1}^{\ell} k_{m}\left(\frac{k_{m}}{2}\left\|\Delta^{-\frac{1}{2}} d_{t} e^{m}\right\|_{L^{2}}^{2}+\frac{\gamma_{1} \varepsilon k_{m}^{\beta}}{2}\left\|\nabla e^{m}\right\|_{L^{2}}^{2}\right) \\
& \leq \tilde{c}_{1} k_{0}^{2-\beta} \rho_{2}(\varepsilon) \exp \left(\tilde{c}_{2} t_{\ell}\right) .
\end{aligned}
$$

We employ the following necessary criterion

$$
k_{0}^{2+4 \frac{(4-N) \delta}{8-(4-N) \delta}} \varepsilon^{-\left\{\frac{\left(2 \sigma_{1}+1\right)(4-N) \delta}{8-(4-N) \delta}+2\left(\sigma_{1}+2\right)\right\}}\left[\tilde{\rho}_{1}(\varepsilon)\right]^{\frac{2 N \delta}{8-(4-N) \delta}} \leq \frac{\tilde{c}_{1}}{2} k_{0}^{2-\beta} \rho_{2}(\varepsilon),
$$

which implies the third condition in (59).

Finally, we need to make sure that

$$
\begin{aligned}
& \sum_{m=1}^{\ell} k_{m}\left[\varepsilon k_{m}^{\beta}\right]^{-\frac{8+(4-N) \delta}{8-(4-N) \delta}}\left[\tilde{\rho}_{1}(\varepsilon)\right]^{\frac{2 N \delta}{8-(4-N) \delta}}\left[k_{0}^{2-\beta} \rho_{2}(\varepsilon)\right]^{1+\frac{(4-N) \delta}{8-(4-N) \delta}} \\
& \quad \leq \frac{\tilde{c}_{1}}{2} k_{0}^{2-\beta} \rho_{2}(\varepsilon) \exp \left(\tilde{c}_{2} t_{\ell+1}\right) .
\end{aligned}
$$

This completes the induction argument and the proof, too.

\section{Error analysis for a fully discrete mixed finite element approximation}

In this section we analyze the fully discrete mixed finite element method (10)-(11) for (25)-(26) on the meshes $J_{k}^{1}$ and $J_{k}^{2}$. Throughout this section, we assume that $u_{0} \in H^{2}(\Omega)$ and $\partial \Omega$ is of class $C^{0,1}$, and that $\left(\mathrm{GA}_{1}\right)-\left(\mathrm{GA}_{3}\right)$ are satisfied.

We recall that the fully discrete mixed finite element discretization of (25)-(26) is defined as: Find $\left\{\left(U^{m}, W^{m}\right)\right\}_{m=1}^{M} \in\left[\mathcal{S}_{h}\right]^{2}$ such that

$$
\begin{array}{cc}
\left(d_{t} U^{m+1}, \eta_{h}\right)+\left(\nabla W^{m+1}, \nabla \eta_{h}\right)=0 & \forall \eta_{h} \in \mathcal{S}_{h}, \\
\varepsilon\left(\nabla U^{m+1}, \nabla v_{h}\right)+\frac{1}{\varepsilon}\left(f\left(U^{m+1}\right), v_{h}\right)=\left(W^{m+1}, v_{h}\right) & \forall v_{h} \in \mathcal{S}_{h},
\end{array}
$$

with some suitable starting value $U^{0}$, and a quasi-uniform "triangulation" $\mathcal{T}_{h}$ of $\Omega$. Where $\mathcal{S}_{h}$ denotes the $P_{1}$ conforming finite element space defined by

$$
\mathcal{S}_{h}:=\left\{v_{h} \in C(\bar{\Omega}) ;\left.v_{h}\right|_{K} \in P_{1}(K), \forall K \in \mathcal{T}_{h}\right\} .
$$

The mixed finite element space $\mathcal{S}_{h} \times \mathcal{S}_{h}$ is the lowest order element among a family of stable mixed finite element spaces known as the Ciarlet-Raviart 
mixed finite elements for the biharmonic problem (cf. $[18,38]$ ), that is, the following inf-sup condition holds

$$
\inf _{0 \neq \eta_{h} \in \mathcal{S}_{h},\left(\eta_{h}, 1\right)=0} \sup _{0 \neq \psi_{h} \in \mathcal{S}_{h}} \frac{\left(\nabla \psi_{h}, \nabla \eta_{h}\right)}{\left\|\psi_{h}\right\|_{H^{1}}\left\|\eta_{h}\right\|_{H^{1}}} \geq c_{0}
$$

for some $c_{0}>0$.

Also, we note that $\left(d_{t} U^{m+1}, 1\right)=0$, which implies that $\left(U^{m+1}, 1\right)=$ $\left(U^{0}, 1\right)$ for $m=0,1, \cdot, M-1$. Hence, the mass is also conserved by the fully discrete solution at each time step.

We define the $L^{2}(\Omega)$-projection $Q_{h}: L^{2}(\Omega) \rightarrow \mathcal{S}_{h}$ by

$$
\left(Q_{h} v-v, \eta_{h}\right)=0 \quad \forall \eta \in \mathcal{S}_{h},
$$

and the elliptic projection $P_{h}: H^{1}(\Omega) \rightarrow \mathcal{S}_{h}$ by

$$
\left(\nabla\left[P_{h} v-v\right], \nabla \eta_{h}\right)=0 \quad \forall \eta_{h} \in \mathcal{S}_{h}, \quad\left(P_{h} v-v, 1\right)=0 .
$$

We refer to Section 4 of [28] for a list of approximation properties of $P_{h}$. In the sequel, we confine to meshes $\mathcal{T}_{h}$ that allow for $H^{1}$-stability of $Q_{h}$, see [14] for their further characterization.

We also introduce space notation $\stackrel{\circ}{\mathcal{S}}_{h}:=\left\{v_{h} \in \mathcal{S}_{h} ;\left(v_{h}, 1\right)=0\right\}$,

and define the discrete inverse Laplace operator: $-\Delta_{h}^{-1}: L_{0}^{2}(\Omega) \rightarrow \stackrel{\circ}{\mathcal{S}}_{h}$ such that

$$
\left(\nabla\left(-\Delta_{h}^{-1} v\right), \nabla \eta_{h}\right)=\left(v, \eta_{h}\right) \quad \forall \eta_{h} \in \mathcal{S}_{h} .
$$

Lemma 4 For $J_{k}=J_{k}^{1}$ or $J_{k}^{2}$, the solution $\left\{\left(U^{m}, W^{m}\right)\right\}_{m=0}^{M}$ of (65)-(66) satisfies

(i) $\frac{1}{|\Omega|} \int_{\Omega} U^{m} \mathrm{~d} x=\frac{1}{|\Omega|} \int_{\Omega} U^{0} \mathrm{~d} x, \quad m=1,2, \ldots, M$,

(ii) $\left\|d_{t} U^{m}\right\|_{H^{-1}} \leq C\left\|\nabla W^{m}\right\|_{L^{2}}, \quad m=1,2, \ldots, M$,

(iii) $\max _{0 \leq m \leq M}\left\{\varepsilon\left\|\nabla U^{m}\right\|_{L^{2}}^{2}+\frac{1}{\varepsilon}\left\|F\left(U^{m}\right)\right\|_{L^{1}}\right\}$

$$
+\sum_{m=1}^{M} k_{m}\left(\left\|\nabla W^{m}\right\|_{L^{2}}^{2}+\varepsilon k_{m}\left\|\nabla d_{t} U^{m}\right\|_{L^{2}}^{2}\right) \leq C \mathcal{J}_{\varepsilon}\left(U^{0}\right),
$$

$$
\sum_{m=1}^{M} k_{m}\left\|d_{t} U^{m}\right\|_{H^{-1}}^{2} \leq C \mathcal{J}_{\varepsilon}\left(U^{0}\right) .
$$

We refer to [27] for a detailed proof of the lemma. 
Remark In view of (i) of Lemma 1 and (i) of Lemma 4, in order for the scheme (65)-(66) to conserve the mass of the underlying physical problem, it is necessary to require $\left(U^{0}-u_{0}^{\varepsilon}, 1\right)=0$ for the starting value $U^{0}$. This condition will be assumed in the rest of this section.

The subsequent step in our error analysis for (65)-(66) is to validate a discrete version of Proposition 1. We define for $\varphi^{m} \in L_{0}^{2}(\Omega) \cap H^{2}(\Omega)$, $0 \leq m \leq M$, and $c=c(\Omega)$,

$$
\begin{aligned}
\hat{C}_{1}\left(\varepsilon,\left\{\varphi^{m}\right\}_{m=0}^{M}\right) & \equiv \max _{J_{k}^{2}}\left\|\varphi^{m}\right\|_{L^{\infty}} \leq c \max _{J_{k}^{2}}\left(\left\|\nabla \varphi^{m}\right\|^{\frac{5-N}{4}}\left\|\Delta \varphi^{m}\right\|_{L^{2}}^{\frac{N-1}{4}}+\left\|\varphi^{m}\right\|_{L^{2}}\right), \\
C_{1}(\varepsilon) & \equiv \max \left\{\hat{C}_{1}\left(\varepsilon,\left\{u^{m}\right\}_{m=0}^{M}\right), \hat{C}_{1}\left(\varepsilon,\left\{u\left(t_{m}\right)\right\}_{m=0}^{M}\right)\right\} \\
& \leq \rho_{7}(\varepsilon, N):=c \varepsilon^{-\left(2 \sigma_{1}+1\right) \frac{5-N}{8}}\left[\tilde{\rho}_{1}(\varepsilon)\right]^{\frac{N-1}{8}},
\end{aligned}
$$

$$
C_{2}(\varepsilon)=\max _{|v| \leq 2 C_{1}(\varepsilon)}\left|f^{\prime \prime}(v)\right|,
$$

and $C_{3}$ be the smallest positive $\varepsilon$-independent constant such that (cf. Chapter 7 of [10])

$$
\begin{aligned}
\max _{J_{k}^{2}}\left\|u^{m}-P_{h} u^{m}\right\|_{L^{\infty}\left(J ; L^{\infty}\right)} & \leq C_{3} h^{\frac{4-N}{2}}|\ln h|^{\frac{3-N}{2}} \max _{J_{k}^{2}}\left\|u^{m}\right\|_{H^{2}} \\
& \leq C_{3} h^{\frac{4-N}{2}}|\ln h|^{\frac{3-N}{2}}\left[\tilde{\rho}_{1}(\varepsilon)\right]^{\frac{1}{2}} .
\end{aligned}
$$

Proposition 2 Let the assumptions of Proposition 1 hold, and $C_{0}$ and $\varepsilon_{0}$ be same as there. Let $\left\{u^{m}\right\}_{m=0}^{M}$ be the solution of (25)-(26) and $\left\{P_{h} u^{m}\right\}_{m=0}^{M}$ be its elliptic projection. Suppose that the assumptions of Theorem 2 are valid. Then there holds for $\varepsilon \in\left(0, \varepsilon_{0}\right)$ and $0 \leq m \leq M$

$$
\begin{aligned}
\lambda_{C H}^{h, k_{0}} & \equiv \inf _{\substack{0 \neq \psi \in L_{0}^{2}(\Omega) \\
\Delta w=\psi, \frac{\partial w}{\partial n}=0}} \frac{\varepsilon\|\nabla \psi\|_{L^{2}}^{2}+\frac{1-\varepsilon}{\varepsilon}\left(f^{\prime}\left(P_{h} u^{m}\right) \psi, \psi\right)}{\|\nabla w\|_{L^{2}}^{2}} \\
& \geq-(1-\varepsilon)\left(C_{0}+1\right),
\end{aligned}
$$

provided that $k_{0}$ and $h$ satisfy for some $c=c(\Omega)$

$$
\begin{aligned}
h^{\frac{4-N}{2}}|\ln h|^{\frac{3-N}{2}} & \leq\left(C_{2}(\varepsilon) C_{3}\left[\rho_{1}(\varepsilon)\right]^{\frac{1}{2}}\right)^{-1} \varepsilon^{2} \\
k_{0} & \leq\left\{\varepsilon^{2\left(\sigma_{1}+1\right)}\left[\rho_{2}(\varepsilon)\right]^{-1}\left(\frac{\varepsilon^{2}}{c}\left[\tilde{\rho}_{1}(\varepsilon)\right]^{-\frac{N}{8}}\right)^{\frac{16}{4-N}}\right\}^{\frac{1}{2-\beta}}
\end{aligned}
$$

Proof. Step 1 From the definitions of $C_{2}(\varepsilon)$ and $C_{3}$, we immediately have $\max _{J_{k}^{2}}\left\|P_{h} u^{m}\right\|_{L^{\infty}} \leq \max _{J_{k}^{2}}\left\{\left\|u^{m}\right\|_{L^{\infty}}+\left\|P_{h} u^{m}-u^{m}\right\|_{L^{\infty}}\right\} \leq 2 \max _{J_{k}^{2}}\left\|u^{m}\right\|_{L^{\infty}}$ 
if $h$ satisfies (73). It then follows from the Mean Value Theorem that

$$
\begin{aligned}
\max _{J_{k}^{2}}\left\|f^{\prime}\left(P_{h} u^{m}\right)-f^{\prime}\left(u^{m}\right)\right\|_{L^{\infty}} & \leq \sup _{|\xi| \leq 2 C_{1}(\varepsilon)}\left|f^{\prime \prime}(\xi)\right| \max _{J_{k}^{2}}\left\|P_{h} u^{m}-u^{m}\right\|_{L^{\infty}} \\
& \leq C_{2}(\varepsilon) C_{3} h^{\frac{4-N}{2}}|\ln h|^{\frac{3-N}{2}}\left[\tilde{\rho}_{1}(\varepsilon)\right]^{\frac{1}{2}} \leq \varepsilon^{2} .
\end{aligned}
$$

Using the inequality $a \geq b-|a-b|$ and (75) we get

$$
f^{\prime}\left(P_{h} u^{m}\right) \geq f^{\prime}\left(u^{m}\right)-\left\|f^{\prime}\left(P_{h} u^{m}\right)-f^{\prime}\left(u^{m}\right)\right\|_{L^{\infty}} \geq f^{\prime}\left(u^{m}\right)-\varepsilon^{2} .
$$

Step 2 By a Gagliardo-Nirenberg's inequality we get for any $0 \leq m \leq M$

(76) $\left\|u\left(t_{m}\right)-u^{m}\right\|_{L^{\infty}} \leq c\left\|u\left(t_{m}\right)-u^{m}\right\|_{L^{2}}^{\frac{4-N}{4}}\left\{\left\|\Delta u\left(t_{m}\right)\right\|_{L^{2}}+\left\|\Delta u^{m}\right\|_{L^{2}}\right\}^{\frac{N}{4}}$

$$
\begin{aligned}
& \leq c\left\|u\left(t_{m}\right)-u^{m}\right\|_{H^{-1}}^{\frac{4-N}{8}}\left\{\left\|\nabla u\left(t_{m}\right)\right\|_{L^{2}}+\left\|\nabla u^{m}\right\|_{L^{2}}\right\}^{\frac{4-N}{8}}\left[\tilde{\rho}_{1}(\varepsilon)\right]^{\frac{N}{8}} \\
& \leq c\left[\tilde{\rho}_{1}(\varepsilon)\right]^{\frac{N}{8}}\left[\varepsilon^{-\left(2 \sigma_{1}+1\right)} \rho_{2}(\varepsilon) k_{0}^{2-\beta}\right]^{\frac{4-N}{16}},
\end{aligned}
$$

thanks to Theorem 2.

If $k_{0}$ satisfies (74), we find by the Mean Value Theorem and (77) that

$$
\begin{aligned}
& \max _{J_{k}^{2}}\left\|f^{\prime}\left(u^{m}\right)-f^{\prime}\left(u\left(t_{m}\right)\right)\right\|_{L^{\infty}} \leq \sup _{|\tilde{\xi}| \leq 2 C_{1}(\varepsilon)}\left|f^{\prime \prime}(\tilde{\xi})\right| \max _{J_{k}^{2}}\left\|u^{m}-u\left(t_{m}\right)\right\|_{L^{\infty}} \\
& \quad \leq c C_{2}(\varepsilon)\left[\tilde{\rho}_{1}(\varepsilon)\right]^{\frac{N}{8}}\left[\varepsilon^{-\left(2 \sigma_{1}+1\right)} \rho_{2}(\varepsilon) k_{0}^{2-\beta}\right]^{\frac{4-N}{16}} \leq \varepsilon^{2},
\end{aligned}
$$

which implies that

$$
f^{\prime}\left(u^{m}\right) \geq f^{\prime}\left(u\left(t_{m}\right)\right)-\varepsilon^{2} .
$$

It follows from (77) and (77) that

$$
f^{\prime}\left(P_{h} u^{m}\right) \geq f^{\prime}\left(u\left(t_{m}\right)\right)-2 \varepsilon^{2} .
$$

In addition, since $\frac{\partial w}{\partial n}=0$ on $\partial \Omega$ we have

$$
\|\psi\|_{L^{2}}^{2}=(\nabla \psi, \nabla w) \leq \frac{1}{2}\left(\frac{\varepsilon}{1-\varepsilon}\|\nabla \psi\|_{L^{2}}^{2}+\frac{1-\varepsilon}{\varepsilon}\|\nabla w\|_{L^{2}}^{2}\right) .
$$

Substituting (78) and (79) into the definition of $\lambda_{C H}^{h, k_{0}}$ we get

$$
\lambda_{C H}^{h, k_{0}} \geq \inf _{\substack{0 \neq \psi \in L_{0}^{2}(\Omega) \\ \Delta w=\psi, \frac{\partial w}{\partial n}=0}} \frac{(1-\varepsilon)\left[\varepsilon\|\nabla \psi\|_{L^{2}}^{2}+\frac{1}{\varepsilon}\left(f^{\prime}\left(u^{m}\right) \psi, \psi\right)\right]}{\|\nabla w\|_{L^{2}}^{2}}-(1-\varepsilon)^{2} .
$$

The proof is completed by applying Proposition 1 .

The main result in this section is stated in the following theorem. 
Theorem 3 Let $\left\{\left(U^{m}, W^{m}\right)\right\}_{m=0}^{M}$ solve (65)-(66) on $J_{k}=J_{k}^{2}$ and on a quasiuniform triangulation $\mathcal{T}_{h}$ of $\Omega$, allowing for inverse inequalities and $H^{1}$ stability of the $L^{2}$-projection in the continuous linear finite element space. Suppose that the assumptions and notation of Theorem 2 hold. For $0<\beta$ $<\frac{1}{2}, N=2,3$, and $v>0$, define

$$
\begin{aligned}
\rho_{7}(\varepsilon, N):=c \varepsilon^{-\left(2 \sigma_{1}+1\right) \frac{5-N}{8}}\left[\tilde{\rho}_{1}(\varepsilon)\right]^{\frac{N-1}{8}}, \\
\gamma_{1}^{*}:=\max \left\{1-\varepsilon^{r}, \gamma_{1}\right\}, \quad \text { for any } r>1, \\
\pi\left(h, k_{0}, \varepsilon, N\right):=h^{4}\left\{h^{2 v}+\ln \left(\frac{1}{k_{0}}\right) \tilde{\rho}_{1}(\varepsilon) h^{2}\right. \\
\left.\quad+\frac{2(1-\varepsilon)}{1-\varepsilon-\gamma_{1}^{\star}} \varepsilon^{-\left(2 \sigma_{1}+6\right)}\left[\rho_{7}(\varepsilon, N)\right]^{2(p-2)}\right\} .
\end{aligned}
$$

Let $k_{0}, h$ and $U^{0}$ satisfy the following constraints

$$
\begin{aligned}
& \text { 1) } k_{0} \leq \tilde{C} \min \left\{\varepsilon^{\frac{3}{\beta}}, \varepsilon^{\frac{\alpha_{0}}{2}},\left[\rho_{3}(\varepsilon, N)\right]^{\tilde{\rho}_{4}(N, \beta)},\left[\rho_{5}(\varepsilon, N)\right]^{\tilde{\rho}_{6}(N, \beta)}\right\}, \\
& \text { 2) } k_{0} \leq\left\{\varepsilon^{2\left(\sigma_{1}+1\right)}\left[\rho_{2}(\varepsilon)\right]^{-1}\left(\frac{\varepsilon^{2}}{c}\left[\tilde{\rho}_{1}(\varepsilon)\right]^{-\frac{N}{8}}\right)^{\frac{16}{4-N}}\right\}^{\frac{1}{2-\beta}}, \\
& \text { 3) } h^{\frac{4-N}{2}}|\ln h|^{\frac{3-N}{2}} \leq\left(C_{2}(\varepsilon) C_{3}\left[\rho_{1}(\varepsilon)\right]^{\frac{1}{2}}\right)^{-1} \varepsilon^{2}, \\
& \text { 4) } k_{0}^{-2 \beta \frac{8+(4-N) \delta}{8-(4-N) \delta}}\left(\pi\left(h, k_{0}, \varepsilon, N\right)+k_{0}^{2-\beta} \rho_{2}(\varepsilon)\right)^{\frac{(4-N) \delta}{8-(4-N) \delta}} \\
& \quad \leq\left(\varepsilon\left[\tilde{\rho}_{1}(\varepsilon)\right]^{\frac{2 N \delta}{8-(4-N) \delta}}\right)^{-1},
\end{aligned}
$$

5) $\left(U^{0}, 1\right)=\left(u_{0}, 1\right)$ and $\left\|U^{0}-u_{0}\right\|_{H^{-1}} \leq C h^{2+v}\left\|u_{0}\right\|_{H^{2}}$.

Then the solution of (65)-(66) satisfies the error estimates, for $\mathcal{E}^{m}:=u\left(t_{m}\right)-$ $U^{m}$,

$$
\begin{aligned}
& \max _{0 \leq m \leq M}\left\|\mathcal{E}^{m}\right\|_{H^{-1}}+\left(\sum_{m=1}^{M} k_{m}^{2}\left\|d_{t} \mathcal{E}^{m}\right\|_{H^{-1}}^{2}\right)^{\frac{1}{2}} \\
& +\left(\sum_{m=1}^{M} k_{m}\left\|\mathcal{E}^{m}\right\|_{L^{2}}^{2}\right)^{\frac{1}{2}} \leq C\left(h^{4} \varepsilon^{-\left(2 \sigma_{1}+3\right)}+\pi\left(h, k_{0}, \varepsilon, N\right)+k_{0}^{(2-\beta)} \rho_{2}(\varepsilon)\right)^{\frac{1}{2}} \\
& \left(\sum_{m=1}^{M} k_{m}\left\|\nabla \mathcal{E}^{m}\right\|_{L^{2}}^{2}\right)^{\frac{1}{2}} \leq C\left(h^{2} \varepsilon^{-\left(2 \sigma_{1}+4\right)}+\pi\left(h, k_{0}, \varepsilon, N\right)+k_{0}^{(2-\beta)} \rho_{2}(\varepsilon)\right)^{\frac{1}{2}} .
\end{aligned}
$$

Proof. As in the proof of Theorem 2, here we divide the proof into four steps. Also, in the proof we make use of the facts $\mathcal{S}_{h} \subset H^{1}(\Omega)$ and $u^{m}-U^{m} \in$ $L_{0}^{2}(\Omega)$. New difficulties that enter the analysis are (i) a different treatment of the super-quadratic error term is used: instead of interpolating $L^{2+\delta}(\Omega)$ 
between $L^{2}(\Omega)$ and $H^{2}(\Omega)$, we carry this out elementwise. (ii) the discrete spectrum result of Proposition 2 is in place of that of Proposition 1.

Steps $1 \& 2$ : Let $E^{m}:=u^{m}-U^{m}$ and $G^{m}:=w^{m}-W^{m}$. We subtract (65)-(66) from (25)-(26) to get the error equations

(81) $\varepsilon\left(\nabla E^{m}, \nabla v_{h}\right)+\frac{1}{\varepsilon}\left(f\left(u^{m}\right)-f\left(U^{m}\right), v_{h}\right)=\left(G^{m}, v_{h}\right) \quad \forall v_{h} \in \mathcal{S}_{h}$.

Introduce the decompositions: $E^{m}:=\Theta^{m}+\Phi^{m}$ and $G^{m}:=\Lambda^{m}+\Psi^{m}$, where

$$
\begin{array}{ll}
\Theta^{m}:=u^{m}-P_{h} u^{m}, & \Phi^{m}:=P_{h} u^{m}-U^{m}, \\
\Lambda^{m}:=w^{m}-P_{h} w^{m}, & \Psi^{m}:=P_{h} w^{m}-W^{m} .
\end{array}
$$

Then from the definition of $P_{h}$ in (68) we can rewrite (80)-(81) as follows

$$
\begin{aligned}
& (82)\left(d_{t} \Phi^{m}, \eta_{h}\right)+\left(\nabla \Psi^{m}, \nabla \eta_{h}\right)=-\left(d_{t} \Theta^{m}, \eta_{h}\right) \text {, } \\
& \text { (83) } \varepsilon\left(\nabla \Phi^{m}, \nabla v_{h}\right)+\frac{1}{\varepsilon}\left(f\left(P_{h} u^{m}\right)-f\left(U^{m}\right), v_{h}\right)=\left(\Psi^{m}, v_{h}\right)+\left(\Lambda^{m}, v_{h}\right) \\
& -\frac{1}{\varepsilon}\left(f\left(u^{m}\right)-f\left(P_{h} u^{m}\right), v_{h}\right) \text {. }
\end{aligned}
$$

Since $E^{m}, \Phi^{m} \in L_{0}^{2}(\Omega)$ for $0 \leq m \leq M$, setting $\eta_{h}=-\Delta_{h}^{-1} \Phi^{m}$ in (82) and $v_{h}=\Phi^{m}$ in (83) and taking summation over $m$ from 1 to $\ell(\leq M)$, after adding the equations we arrive at

$$
\begin{aligned}
\frac{1}{2} \| & \nabla \Delta_{h}^{-1} \Phi^{\ell}\left\|_{L^{2}}^{2}+\sum_{m=1}^{\ell} \frac{k_{m}^{2}}{2}\right\| \nabla \Delta_{h}^{-1} d_{t} \Phi^{m} \|_{L^{2}}^{2} \\
& +\sum_{m=1}^{\ell} k_{m}\left(\varepsilon\left\|\nabla \Phi^{m}\right\|_{L^{2}}^{2}+\frac{1}{\varepsilon}\left(f\left(P_{h} u^{m}\right)-f\left(U^{m}\right), \Phi^{m}\right)\right) \\
= & \sum_{m=1}^{\ell} k_{m}\left(-\left(d_{t} \Theta^{m},-\Delta_{h}^{-1} \Phi^{m}\right)+\left(\Lambda^{m}, \Phi^{m}\right)\right) \\
& +\frac{1}{\varepsilon} \sum_{m=1}^{\ell} k_{m}\left(f\left(u^{m}\right)-f\left(P_{h} u^{m}\right), \Phi^{m}\right)+\left\|\nabla \Delta_{h}^{-1} \Phi^{0}\right\|_{L^{2}}^{2} .
\end{aligned}
$$


The first sum on the right hand side can be bounded by taking into account the character of the stretched mesh.

$$
\begin{aligned}
& \sum_{m=1}^{\ell} k_{m}\left\{\left(d_{t} \Theta^{m}, \Delta_{h}^{-1} \Phi^{m}\right)+\left(\Lambda^{m}, \Phi^{m}\right)\right\} \\
& \leq C \sum_{m=1}^{\ell} k_{m}\left\{\ln \left(\frac{1}{k_{0}}\right)\left\|\tilde{d}_{t} \Theta^{m}\right\|_{H^{-1}}^{2}+\frac{2(1-\varepsilon)}{\varepsilon\left(1-\varepsilon-\gamma_{1}^{\star}\right)}\left\|\Lambda^{m}\right\|_{H^{-1}}^{2}\right\} \\
& \quad+\frac{\varepsilon\left(1-\varepsilon-\gamma_{1}^{\star}\right)}{2(1-\varepsilon)} \sum_{m=1}^{\ell} k_{m}\left\|\nabla \Phi^{m}\right\|_{L^{2}}^{2} \\
& \quad+\left[\ln \left(\frac{1}{k_{0}}\right)\right]^{-1} \sum_{m=1}^{\ell} \frac{1}{m}\left\|\nabla \Delta_{h}^{-1} \Phi^{m}\right\|_{L^{2}}^{2}
\end{aligned}
$$

We make use of the following approximation properties in $H^{-1}$ of the elliptic projection $P_{h}$, for $\kappa=0,1$ (cf. [22])

$$
\left\|u^{m}-P_{h} u^{m}\right\|_{H^{-1}} \leq C h^{3-\kappa}\left\|u^{m}\right\|_{H^{2-\kappa}} .
$$

Thanks to Lemma 3 (vii), we can bound the right hand side of (85) by

$$
\begin{gathered}
C\left\{\ln \left(\frac{1}{k_{0}}\right) \tilde{\rho}_{1}(\varepsilon) h^{6}+\frac{2(1-\varepsilon)}{\varepsilon\left(1-\varepsilon-\gamma_{1}^{\star}\right)} h^{6}\right\} \\
+\frac{\varepsilon\left(1-\varepsilon-\gamma_{1}^{\star}\right)}{2(1-\varepsilon)} \sum_{m=1}^{\ell} k_{m}\left\|\nabla \Phi^{m}\right\|_{L^{2}}^{2} \\
+\left[\ln \left(\frac{1}{k_{0}}\right)\right]^{-1} \sum_{m=1}^{\ell} \frac{1}{m}\left\|\nabla \Delta_{h}^{-1} \Phi^{m}\right\|_{L^{2}}^{2} .
\end{gathered}
$$

Because of the inequality at the beginning of the proof of Proposition 2, the second sum on the right hand side of (84) can be bounded by

$$
\begin{aligned}
& \left.\frac{1}{\varepsilon} \sum_{m=1}^{\ell} k_{m}\left(f\left(u^{m}\right)\right)-f\left(P_{h} u^{m}\right), \Phi^{m}\right)=\frac{1}{\varepsilon} \sum_{m=1}^{\ell} k_{m}\left(f^{\prime}\left(\xi^{m}\right) \Theta^{m}, \Phi^{m}\right) \\
& \leq \sum_{m=1}^{\ell} k_{m}\left\{\frac{\varepsilon\left(1-\varepsilon-\gamma_{1}^{\star}\right)}{2(1-\varepsilon)}\left\|\nabla \Phi^{m}\right\|_{L^{2}}^{2}\right. \\
& \left.\quad+C \frac{2(1-\varepsilon)}{\varepsilon^{3}\left(1-\varepsilon-\gamma_{1}^{\star}\right)}\left\|\Theta^{m}\right\|_{L^{2}}^{2}\left\|f^{\prime}\left(\xi^{m}\right)\right\|_{L^{\infty}}^{2}\right\} \\
& \leq \frac{\varepsilon\left(1-\varepsilon-\gamma_{1}^{\star}\right)}{2(1-\varepsilon)} \sum_{m=1}^{\ell} k_{m}\left\|\nabla \Phi^{m}\right\|_{L^{2}}^{2} \\
& \quad+C h^{4} \frac{2(1-\varepsilon)}{1-\varepsilon-\gamma_{1}^{\star}} \varepsilon^{-\left(2 \sigma_{1}+6\right)}\left[\rho_{7}(\varepsilon, N)\right]^{2(p-2)} .
\end{aligned}
$$


By $\left(\mathrm{GA}_{1}\right)_{3}$, the last term on the left hand side of (84) is bounded from below by

$$
\begin{aligned}
& \frac{1}{\varepsilon} \sum_{m=1}^{\ell} k_{m}\left(f\left(P_{h} u^{m}\right)-f\left(U^{m}\right), \Phi^{m}\right) \\
& \quad \geq \frac{1}{\varepsilon} \sum_{m=1}^{\ell} k_{m}\left(\gamma_{1}\left(f^{\prime}\left(P_{h} u^{m}\right) \Phi^{m}, \Phi^{m}\right)-\gamma_{2}\left\|\Phi^{m}\right\|_{L^{2+\delta}}^{2+\delta}\right) .
\end{aligned}
$$

Substituting (85)-(88) into (84) we arrive at

$$
\begin{aligned}
& \frac{1}{2}\left\|\nabla \Delta_{h}^{-1} \Phi^{\ell}\right\|_{L^{2}}^{2}+\sum_{m=1}^{\ell} \frac{k_{m}^{2}}{2}\left\|\nabla \Delta_{h}^{-1} d_{t} \Phi^{m}\right\|_{L^{2}}^{2} \\
& \quad+\frac{\gamma_{1}}{1-\varepsilon} \sum_{m=1}^{\ell} k_{m}\left(\varepsilon\left\|\nabla \Phi^{m}\right\|_{L^{2}}^{2}+\frac{1-\varepsilon}{\varepsilon}\left(f^{\prime}\left(P_{h} u^{m}\right) \Phi^{m}, \Phi^{m}\right)\right) \\
& \leq C \pi\left(h, k_{0}, \varepsilon, N\right)+\frac{\gamma_{2}}{\varepsilon} \sum_{m=1}^{\ell} k_{m}\left\|\Phi^{m}\right\|_{L^{2+\delta}}^{2+\delta} \\
& \quad+\left[\ln \left(\frac{1}{k_{0}}\right)\right]^{-1} \sum_{m=1}^{\ell} \frac{1}{m}\left\|\nabla \Delta_{h}^{-1} \Phi^{m}\right\|_{L^{2}}^{2} .
\end{aligned}
$$

We could bound the last term on the left hand side from below using Proposition 2, however, this will consume all the contribution of $\varepsilon\left\|\nabla \Phi^{m}\right\|_{L^{2}}^{2}$ on the left hand side. On the other hand, in order to bound the super-quadratic term on the right hand side in Step 3 below, we do need some (small) help from this $\varepsilon\left\|\nabla \Phi^{m}\right\|_{L^{2}}^{2}$. For that reason, we only apply Proposition 2 with a scaling factor $\left(1-\frac{k^{\beta}}{2}\right)$,

$$
\begin{aligned}
& \left(1-\frac{k^{\beta}}{2}\right)\left(\varepsilon\left\|\nabla \Phi^{m}\right\|_{L^{2}}^{2}+\frac{1-\varepsilon}{\varepsilon}\left(f^{\prime}\left(P_{h} u^{m}\right) \Phi^{m}, \Phi^{m}\right)\right) \\
& \geq-\left(1-\frac{k^{\beta}}{2}\right)(1-\varepsilon)\left(C_{0}+2\right)\left\|\nabla \Delta^{-1} \Phi^{m}\right\|_{L^{2}}^{2} \\
& \geq-\left(C_{0}+1\right)\left\|\nabla \Delta^{-1} \Phi^{m}\right\|_{L^{2}}^{2} .
\end{aligned}
$$

The leftover term is controlled by

$$
\frac{\gamma_{1} k^{\beta}}{\varepsilon}\left(f^{\prime}\left(P_{h} u^{m}\right) \Phi^{m}, \Phi^{m}\right) \leq \frac{\gamma_{1} \varepsilon k^{\beta}}{4}\left\|\nabla \Phi^{m}\right\|_{L^{2}}^{2}+\tilde{c}_{0} \frac{\gamma_{1} k^{\beta}}{\varepsilon^{3}}\left\|\nabla \Delta_{h}^{-1} \Phi^{m}\right\|_{L^{2}}^{2}
$$


Inserting these results into (89), we finally get

(90)

$$
\begin{aligned}
& \frac{1}{2}\left\|\nabla \Delta_{h}^{-1} \Phi^{\ell}\right\|_{L^{2}}^{2}+\sum_{m=1}^{\ell} k_{m}\left[\frac{k_{m}}{2}\left\|\nabla \Delta_{h}^{-1} d_{t} \Phi^{m}\right\|_{L^{2}}^{2}+\frac{\gamma_{1} \varepsilon k^{\beta}}{2(1-\varepsilon)}\left\|\nabla \Phi^{m}\right\|_{L^{2}}^{2}\right] \\
& \leq C \pi\left(h, k_{0}, \varepsilon, N\right)+\frac{\gamma_{2}}{\varepsilon} \sum_{m=1}^{\ell} k_{m}\left\|\Phi^{m}\right\|_{L^{2+\delta}}^{2+\delta} \\
& \quad+\left\{\left[\ln \left(\frac{1}{k_{0}}\right)\right]^{-1}+\tilde{c}_{0} \gamma_{1} k^{\beta} \varepsilon^{-3}\right\} \sum_{m=1}^{\ell} \frac{1}{m}\left\|\nabla \Delta_{h}^{-1} \Phi^{m}\right\|_{L^{2}}^{2} .
\end{aligned}
$$

where we have used the fact that $0<\varepsilon<1$ and $\left\|\nabla \Delta^{-1} v_{h}\right\|_{L^{2}}=\left\|\nabla \Delta_{h}^{-1} v_{h}\right\|_{L^{2}}$ for any $v_{h} \in \stackrel{\circ}{\mathcal{S}}_{h}$.

Step 3: Notice that the structure of (91) is exactly the same as in (47). Hence, we can follow the argumentation of Step 3 in the proof of Theorem 1 with the following modification: the estimates (49) and (52) (up to second to the last inequality) are performed for every $K \in \mathcal{T}_{h}$; by convexity of the function $g(s)=s^{q}$, for $q \geq 1$ and $s \geq 0$ we can afterward recover estimates for $\Omega=\bigcup K$ which involves a uniform constant $C$. We refer to Section 3 of [29] for the details of a similar type argument. Finally, we make use of the properties of $-\Delta_{h}^{-1}$ and $\Phi^{m} \in \mathcal{S}_{h}$, for $0 \leq m \leq M$. Consequently, we can reduce (91) into the following form which corresponds to (53),

$$
\begin{aligned}
\frac{1}{2} \| & \nabla \Delta_{h}^{-1} \Phi^{\ell} \|_{L^{2}}^{2}+\sum_{m=1}^{\ell} k_{m}\left\{\frac{k_{m}}{2}\left\|\nabla \Delta_{h}^{-1} d_{t} \Phi^{m}\right\|_{L^{2}}^{2}+\frac{\gamma_{1} \varepsilon k^{\beta}}{2(1-\varepsilon)}\left\|\nabla \Phi^{m}\right\|_{L^{2}}^{2}\right\} \\
\leq & C \pi\left(h, k_{0}, \varepsilon, N\right)+\left\{\left[\ln \left(\frac{1}{k_{0}}\right)\right]^{-1}+\tilde{c}_{0} \gamma_{1} k^{\beta} \varepsilon^{-3}\right\} \\
& \times \sum_{m=1}^{\ell} \frac{1}{m}\left\|\nabla \Delta_{h}^{-1} \Phi^{m}\right\|_{L^{2}}^{2} \\
& +C\left[\tilde{\rho}_{1}(\varepsilon)\right]^{\frac{2 N \delta}{8-(4-N) \delta}} \sum_{m=0}^{\ell} k_{m}\left[\varepsilon k_{m}^{\beta}\right]^{-\frac{8+(4-N) \delta}{8-(4-N) \delta}}\left\|\nabla \Delta_{h}^{-1} \Phi^{m}\right\|_{L^{2}}^{2\left(1+\frac{(4-N) \delta}{8-(4-N) \delta}\right)} \\
& +C k_{0}^{2+4 \frac{(4-N) \delta}{8-(4-N) \delta}} \varepsilon^{-\left\{\frac{\left(2 \sigma_{1}+1\right)(4-N) \delta}{8-(4-N) \delta}+2\left(\sigma_{1}+2\right)\right\}}\left[\tilde{\rho}_{1}(\varepsilon)\right]^{\frac{2 N \delta}{8-(4-N) \delta}} .
\end{aligned}
$$

Step 4: We conclude the proof by an inductive argument based on (91). Suppose that for

$$
k_{0} \leq \tilde{C} \min \left\{\varepsilon^{\frac{3}{\beta}}, \varepsilon^{\frac{\alpha_{0}}{2}},\left[\rho_{3}(\varepsilon)\right]^{\tilde{\rho}_{4}(N, \beta)},\left[\rho_{5}(\varepsilon, N)\right]^{\tilde{\rho}_{6}(N, \beta)}\right\}
$$

and $0<\beta<\frac{1}{2}$, there exist two positive constants

$$
\tilde{c}_{1}=\tilde{c}_{1}\left(t_{\ell}, \Omega, u_{0}, \sigma_{i}, p\right), \quad \tilde{c}_{2}=\tilde{c}_{2}\left(t_{\ell}, \Omega, u_{0}, \sigma_{i}, p ; C_{0}\right),
$$


independent of $k$ and $\varepsilon$, such that the following inequality holds

$$
\begin{aligned}
\max _{0 \leq m \leq \ell} & \frac{1}{2}\left\|\nabla \Delta_{h}^{-1} \Phi^{m}\right\|_{L^{2}}^{2}+\sum_{m=1}^{\ell} k_{m} \\
\times & \left\{\frac{k_{m}}{2}\left\|\nabla \Delta_{h}^{-1} d_{t} \Phi^{m}\right\|_{L^{2}}^{2}+\frac{\gamma_{1} \varepsilon k_{m}^{\beta}}{2}\left\|\nabla \Phi^{m}\right\|_{L^{2}}^{2}\right\} \\
& \leq \tilde{c}_{1}\left(\pi\left(h, k_{0}, \varepsilon, N\right) k_{0}^{2-\beta} \rho_{2}(\varepsilon)\right) \exp \left(c_{2} t_{\ell}\right) .
\end{aligned}
$$

Criterion (64) then gives the third condition in (93).

We also need to make sure that

$$
\begin{aligned}
& \sum_{m=1}^{\ell+1} k_{m}\left[\varepsilon k_{m}^{\beta}\right]^{-\frac{8+(4-N) \delta}{8-(4-N) \delta}}\left[\tilde{\rho}_{1}(\varepsilon)\right]^{\frac{2 N \delta}{8-(4-N) \delta}}\left(\pi\left(h, k_{0}, \varepsilon, N\right)+k_{0}^{2-\beta} \rho_{2}(\varepsilon)\right)^{1+\frac{(4-N) \delta}{8-(4-N) \delta}} \\
& \text { (94) } \leq \frac{\tilde{c}_{1}}{2}\left(\pi\left(h, k_{0}, \varepsilon, N\right)+k_{0}^{2-\beta} \rho_{2}(\varepsilon)\right) \exp \left(c_{2} t_{\ell+1}\right) .
\end{aligned}
$$

This completes the induction proof of (93).

Finally, the assertion (i) follows from applying the triangle inequality on $E^{m}=\Theta^{m}+\Phi^{m}$ and Theorem 2 . The assertion (ii) follows in the same way. The proof is complete.

Remark (a). The proof clearly shows how the three mesh conditions arise. The first entry in condition 1) reflects the subtle interplay between accuracy requirements and smallness of the time-steps $k_{0}$; the second entry is for the stability of the time discretization (see $\left.(\mathrm{GA})_{3}\right)$. The remaining entries in 1) account for the super-quadratic nonlinearity of $f\left(\right.$ see $\left.\left(\mathrm{GA}_{1}\right)_{3}\right)$. The conditions 2) and 3) are to ensure the discrete spectrum estimate (see Proposition 2). A slight coupling of temporal and spatial discretization parameters is given by 4 ), which deteriorates as $\beta$ gets smaller.

(b). Clearly, both $U^{0}=Q_{h} u_{0}$ and $U^{0}=P_{h} u_{0}$ satisfy the condition 5) with $v=1$. The $L^{2}$ projection $Q_{h} u_{0}$ has the advantage of being cheaper to be obtained compared to the elliptic projection $P_{h} u_{0}$. Also, we note that the first identity in 5) is necessary in order for the fully discrete scheme (65)-(66) to conserve the mass.

We conclude this section with a corollary which addresses the case $u_{0} \in$ $H^{3}(\Omega)$ and $\partial \Omega \in C^{2,1}$ by extending Corollary 1 to the fully discrete scheme (65)-(66). Its proof follows the lines of that for Theorem 3.

Corollary 2 Let $\left\{\left(U^{m}, W^{m}\right)\right\}_{m=0}^{M}$ solve (65)-(66) on a quasi-uniform mesh $J_{k}^{1}$ and a quasi-uniform triangulation $\mathcal{T}_{h}$ of $\Omega$. Let the assumptions of Corollary 1 hold and the notation of Theorem 3 be valid. For $0<\beta<\frac{1}{2}, v>0$ 
and $N=2,3$, let

$$
\hat{\pi}(h, \varepsilon, N):=h^{4}\left\{h^{2 v}+\rho_{1}(\varepsilon)+\frac{2(1-\varepsilon)}{1-\varepsilon-\gamma_{1}^{\star}} \varepsilon^{-\left(2 \sigma_{1}+6\right)}\left[\rho_{7}(\varepsilon, N)\right]^{2(p-2)}\right\},
$$

suppose that $k, h$ and $U^{0}$ satisfy the following constraints

1). $k \leq \tilde{C} \min \left\{\varepsilon^{\frac{3}{\beta}}, \varepsilon^{\alpha_{0}},\left[\rho_{3}(\varepsilon, N)\right]^{\tilde{\rho}_{4}(N, \beta)},\left[\rho_{5}(\varepsilon, N)\right]^{\tilde{\rho}_{6}(N, \beta)}\right\}$,

2). $k \leq\left\{\varepsilon^{2\left(\sigma_{1}+1\right)}\left[\rho_{2}(\varepsilon)\right]^{-1}\left(\frac{\varepsilon^{2}}{c}\left[\tilde{\rho}_{1}(\varepsilon)\right]^{-\frac{N}{8}}\right)^{\frac{16}{4-N}}\right\}^{\frac{1}{2-\beta}}$,

3). $h^{\frac{4-N}{2}}|\ln h|^{\frac{3-N}{2}} \leq\left(C_{2}(\varepsilon) C_{3}\left[\rho_{1}(\varepsilon)\right]^{\frac{1}{2}}\right)^{-1} \varepsilon^{2}$,

4). $k^{-\beta \frac{8+(4-N) \delta}{8-(4-N) \delta}}\left(\hat{\pi}(h, \varepsilon, N)+k^{2-\beta} \rho_{2}(\varepsilon)\right)^{\frac{(4-N) \delta}{8-(4-N) \delta}} \leq\left(\varepsilon\left[\tilde{\rho}_{1}(\varepsilon)\right]^{\frac{2 N \delta}{8-(4-N) \delta}}\right)^{-1}$,

5). $\left(U^{0}, 1\right)=\left(u_{0}, 1\right)$ and $\left\|U^{0}-u_{0}\right\|_{H^{-1}} \leq C h^{2+v}\left\|u_{0}\right\|_{H^{2}}$.

Then the solution of (65)-(66) satisfies the error estimates, for $\mathcal{E}^{m}:=u\left(t_{m}\right)-$ $U^{m}$,

(i) $\max _{0 \leq m \leq M}\left\|\mathcal{E}^{m}\right\|_{H^{-1}}+\left(k \sum_{m=1}^{M} k\left\|d_{t} \mathcal{E}^{m}\right\|_{H^{-1}}^{2}\right)^{\frac{1}{2}}+\left(k \sum_{m=1}^{M}\left\|\mathcal{E}^{m}\right\|_{L^{2}}^{2}\right)^{\frac{1}{2}}$

$$
\leq C\left\{h^{4} \varepsilon^{-\left(2 \sigma_{1}+3\right)}+\hat{\pi}(h, \varepsilon, N)+k_{0}^{(2-\beta)} \rho_{2}(\varepsilon)\right\}^{\frac{1}{2}},
$$

(ii)

$$
\begin{aligned}
& \left(k \sum_{m=1}^{M}\left\|\nabla \mathcal{E}^{m}\right\|_{L^{2}}^{2}\right)^{\frac{1}{2}} \\
& \quad \leq C\left\{h^{2} \varepsilon^{-\left(2 \sigma_{1}+4\right)}+\hat{\pi}(h, \varepsilon, N)+k_{0}^{(2-\beta)} \rho_{2}(\varepsilon)\right\}^{\frac{1}{2}} .
\end{aligned}
$$

Acknowledgements. The first author would like to thank Nicholas Alikakos for explaining all the fascinating properties of the Allen-Cahn and Cahn-Hilliard equations to him. He would also like to thank Nicholas Alikakos and Xinfu Chen for answering his questions regarding the spectrum estimate in Proposition 1. The second author gratefully acknowledges financial support by the DFG.

\section{References}

1. Alikakos, N.D., Bates, P.W., Chen, X.: Convergence of the Cahn-Hilliard equation to the Hele-Shaw model. Arch. Rational Mech. Anal. 128(2), 165-205 (1994)

2. Alikakos, N.D., Fusco, G.: The spectrum of the Cahn-Hilliard operator for generic interface in higher space dimensions. Indiana Univ. Math. J. 42(2), 637-674 (1993)

3. Allen, S., Cahn, J.W.: A microscopic theory for antiphase boundary motion and its application to antiphase domain coarsening. Acta Metall. 27, 1084-1095 (1979) 
4. Barrett, J.W., Blowey, J.F.: An error bound for the finite element approximation of a model for phase separation of a multi-component alloy. IMA J. Numer. Anal. 16(2), 257-287 (1996)

5. Barrett, J.W., Blowey, J.F.: Finite element approximation of a model for phase separation of a multi-component alloy with non-smooth free energy. Numer. Math. 77(1), 1-34 (1997)

6. Barrett, J.W., Blowey, J.F., Garcke H.: On fully practical finite element approximations of degenerate Cahn-Hilliard systems. M2AN Math. Model. Numer. Anal. 35(4), 713-748 (2001)

7. Bates, P.W., Fife, P.C.: The dynamics of nucleation for the Cahn-Hilliard equation. SIAM J. Appl. Math. 53(4), 990-1008 (1993)

8. Blowey, J.F., Elliott, C.M.: The Cahn-Hilliard gradient theory for phase separation with nonsmooth free energy, II. Numer. Anal. Eur. J. Appl. Math. 3(2), 147-179 (1992)

9. Blum, H., Rannacher, R.: On the boundary value problem of the biharmonic operator on domains with angular corners. Math. Meth. Appl. Sci. 2(4), 556-581 (1980)

10. Brenner, S.C., Scott, L.R.: The mathematical theory of finite element methods. Springer-Verlag, New York, 1994

11. Cahn, J.W., Elliott, C.M., Novick-Cohen, A.: The Cahn-Hilliard equation with a concentration dependent mobility: motion by minus the Laplacian of the mean curvature. Eur. J. Appl. Math. 7(3), 287-301 (1996)

12. Cahn, J.W., Hilliard, J.E.: Free energy of a nonuniform system I, Interfacial free energy. J. Chem. Phys. 28, 258-267 (1958)

13. Cahn, J.W., Novick-Cohen, A.: Limiting motion for an Allen-Cahn/Cahn-Hilliard system. In: Free boundary problems theory and applications (Zakopane 1995), Longman, Harlow, pp. 89-97, 1996,

14. Carstensen, C.: Merging the Bramble-Pasciak-Steinbach and the Crouzeix-Thomée criterion for $H^{1}$-stability of the $L^{2}$-projection onto finite element spaces. Math. Comp. 71, pp. 157-163 (2002)

15. Chen, X.: Spectrum for the Allen-Cahn Cahn-Hilliard and phase-field equations for generic interfaces. Comm. Partial Diff. Eqs. 19(7-8), 1371-1395 (1994)

16. Chen, X.: Global asymptotic limit of solutions of the Cahn-Hilliard equation. J. Diff. Geom. 44(2), 262-311 (1996)

17. Chen, X., Elliott, C.M., Gardiner A., Zhao J.J.: Convergence of numerical solutions to the Allen-Cahn equation. Appl. Anal. 69(1), 47-56 (1998)

18. Ciarlet, P.G.: The finite element method for elliptic problems. North-Holland Publishing Co. Amsterdam, 1978, Studies in Mathematics and its Applications Vol. 4

19. Copetti, M.I.M., Elliott, C.M.: Numerical analysis of the Cahn-Hilliard equation with a logarithmic free energy. Numer. Math. 63(1), 39-65 (1992)

20. de Mottoni, P., Schatzman, M.: Geometrical evolution of developed interfaces. Trans. Am. Math. Soc. 347(5), 1533-1589 (1995)

21. Du, Q., Nicolaides, R.A.: Numerical analysis of a continuum model of phase transition. SIAM J. Numer. Anal. 28(5) 1310-1322 (1991)

22. Dupont, T.: Some $L^{2}$ error estimates for parabolic Galerkin methods. The mathematical foundations of the finite element method with applications to partial differential equations (Proc. Sympos. Univ. Maryland Baltimore Md. 1972), Academic Press, New York, 491-504 (1972)

23. Elliott, C.M., French D.A.: Numerical studies of the Cahn-Hilliard equation for phase separation. IMA J. Appl. Math. 38(2), 97-128 (1987)

24. Elliott, C.M., French D.A.: A nonconforming finite-element method for the twodimensional Cahn-Hilliard equation. SIAM J. Numer. Anal. 26(4), 884-903 (1989) 
25. Elliott, C.M., French D.A., Milner F.A.: A second order splitting method for the Cahn-Hilliard equation. Numer. Math. 54(5), 575-590 (1989)

26. Elliott, C.M., Songmu Z.: On the Cahn-Hilliard equation. Arch. Rational Mech. Anal. 96(4), 339-357 (1986)

27. Feng, X., Prohl, A.: Numerical analysis of the Cahn-Hilliard equation and approximation for the Hele-Shaw problem Part I: error analysis under minimum regularities. 2001, IMA Technical Report \#1798 downloadable at http://www.ima.umn.edu/preprints/jul01/jul01.html

28. Feng, X., Prohl, A.: Numerical analysis of the Allen-Cahn equation and approximation for mean curvature flows. Numer. Math. 3, 35-65 (2003)

29. Feng, X., Prohl, A.: Numerical analysis of the Cahn-Hilliard equation and approximation for the Hele-Shaw problem Part II: error analysis and convergence of the interface. Interfaces and Free Boundaries (submitted), 2003, IMA Technical Report \#1799 downloadable at http://www.ima.umn.edu/preprints/jul01/jul01.html

30. Feng, X., Prohl, A.: Analysis of a fully discrete finite element method for the phase field model and approximation of its sharp interface limits. Math. Comp. 73, 541-567 (2004)

31. French, D.A., Jensen, S.: Long-time behaviour of arbitrary order continuous time G, alerkin schemes for some one-dimensional phase transition problems. IMA J. Numer. Anal. 14(3), 421-442 (1994)

32. Friedman, A.: Variational principles and free-boundary problems. Robert E. Krieger Publishing Co. Inc. Malabar FL, second edition, 1988

33. Mullins, W.W., Sekerka, J.: Morphological stability of a particle growing by diffusion or heat flow. J. Appl. Math. 34, 322-329 (1963)

34. Novick-Cohen, A.: The Cahn-Hilliard equation: mathematical and modeling perspectives. Adv. Math. Sci. Appl. 8(2), 965-985 (1998)

35. Novick-Cohen, A.: Triple-junction motion for an Allen-Cahn/Cahn-Hilliard system. Phys. D 137(1-2), 1-24 (2000)

36. Pego, R.L.: Front migration in the nonlinear Cahn-Hilliard equation. Proc. Roy. Soc. London Ser. A 422(1863), 261-278 (1989)

37. Prohl, A.: Projection and Quasi-Compressibility Methods for Solving the incompressible Navier-Stokes equations. Advances in Numerical Mathematics, B.G. Teubner-Verlag, Stuttgart, 1997

38. Scholz R.: A mixed method for 4 th order problems using linear finite elements. RAIRO Anal. Numér. 12(1), 85-90 (1978)

39. Stoth, B.: Convergence of the Cahn-Hilliard equation to the Mullins-Sekerka problem in spherical symmetry. J. Diff. Eqs. 125(1), 154-183 (1996) 